US Army Corps

of Engineers $\mathrm{B}_{\circledast}$

Engineer Research and

Development Center

\title{
Simulated Aging and Characterization of Phase Change Materials for Thermal Management of Building Envelopes
}

Elizabeth J. Gao, Jignesh Patel, Veera M. Boddu, L.D. Stephenson, ～September 2015 Debbie Lawrence, and Ashok Kumar
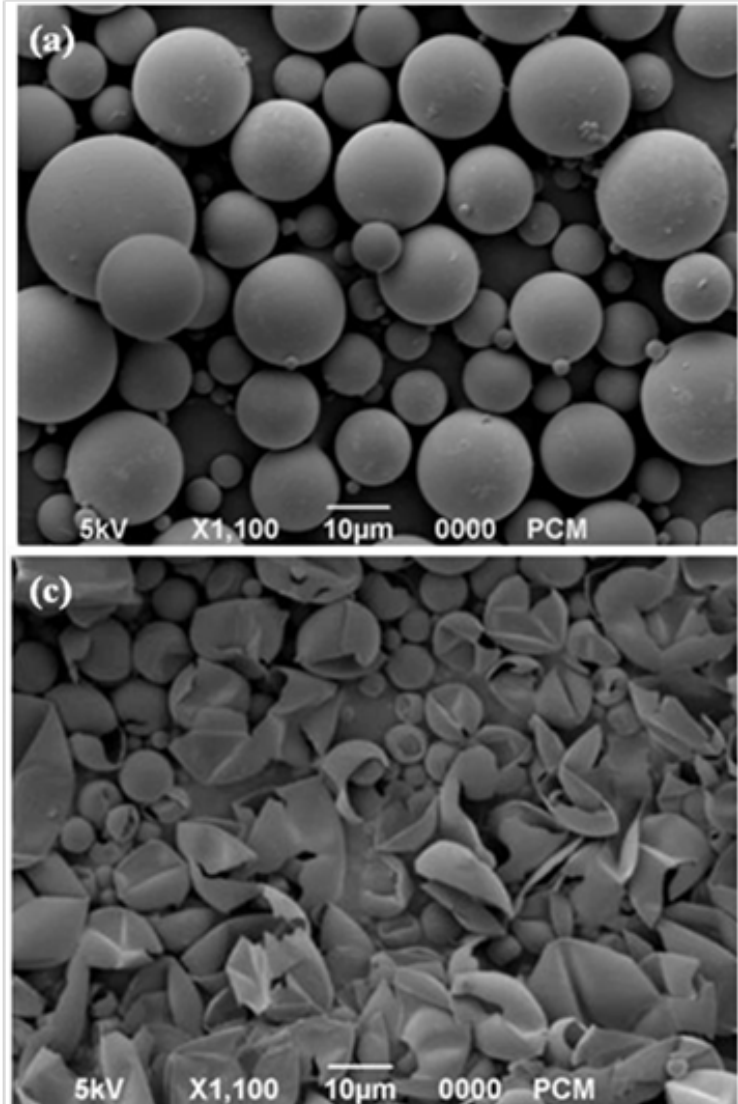
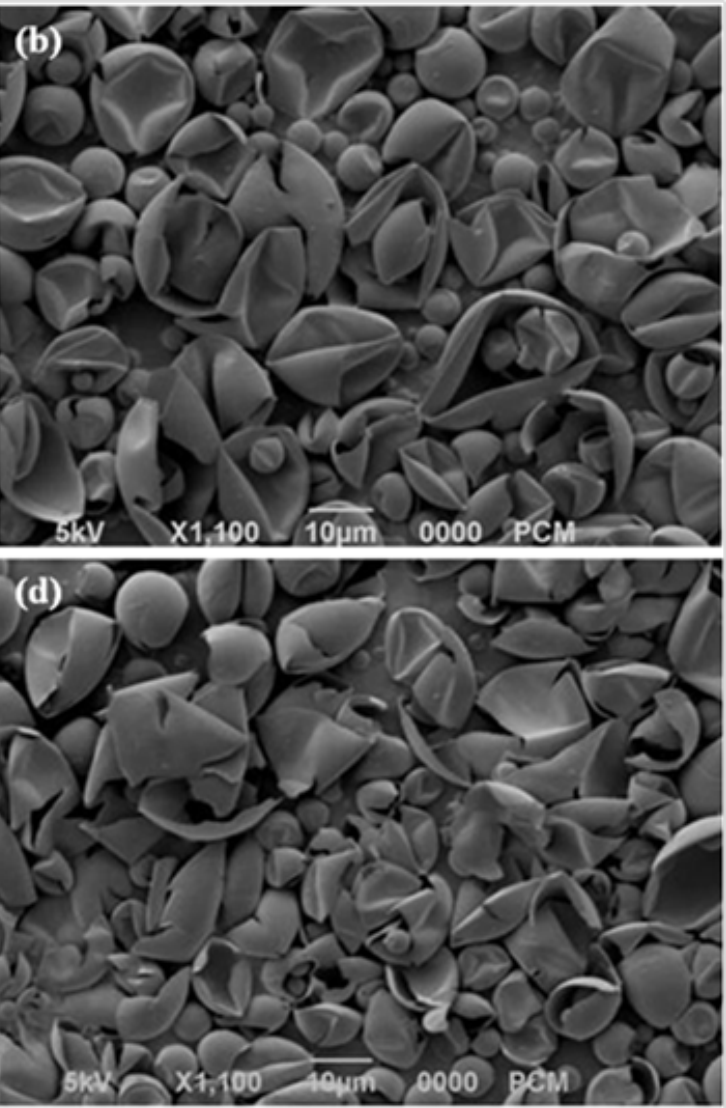
The US Army Engineer Research and Development Center (ERDC) solves the nation's toughest engineering and environmental challenges. ERDC develops innovative solutions in civil and military engineering, geospatial sciences, water resources, and environmental sciences for the Army, the Department of Defense, civilian agencies, and our nation's public good. Find out more at www.erdc.usace.army.mil.

To search for other technical reports published by ERDC, visit the ERDC online library at http://acwc.sdp.sirsi.net/client/default. 


\section{Simulated Aging and Characterization of Phase Change Materials for Thermal Management of Building Envelopes}

Elizabeth J. Gao, Jignesh Patel, Veera M. Boddu, L.D. Stephenson, Debbie Lawrence, and Ashok Kumar

Construction Engineering Research Laboratory (CERL)

U.S. Army Engineer Research and Development Center 2902 Newmark Dr.

Champaign, IL 61822-1076

Final Report

Approved for public release; distribution is unlimited.

Prepared for Headquarters, U.S. Army Corps of Engineers

Washington, DC 20314-1000 


\section{Abstract}

Because phase change materials (PCMs) can absorb or release heat when they undergo phase changes (solid to liquid, liquid to solid, or solid to gas), they are considered especially promising candidates for use in heating and cooling applications for building envelopes. This work investigated the stability of four commercially available PCMs, and developed an accelerated testing protocol to simulate the long-term performance of PCMs in an operational scenario. The selected PCM s were subjected to up to 5,400 cycles of thermal cycling at 90 minutes per cycle, over wide temperature ranges to simulate 20 years of use in building envelopes. At 3-4 week time intervals, the samples were taken from the thermal cycling chamber and Scanning Electron Microscope (SEM) micrographs, Differential scanning calorimeter (DSC), Thermogravimetric Analysis (TGA), and Fourier Transform Infrared Spectroscopy (FTIR) data were obtained and compared to baseline (pre-cycling data). Results indicated that baseline latent heat values and freezing and melting transition temperatures were generally in accordance with the manufacturer's stated values. All PCM materials evaluated tended to lose some latent heat storage capacity as they underwent thermal cycling. 


\section{Contents}

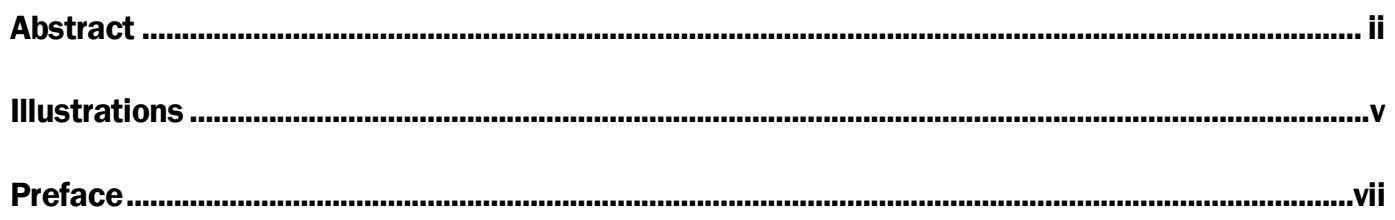

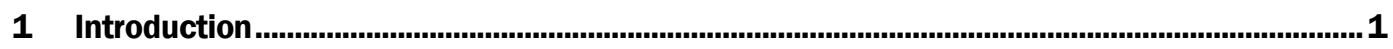

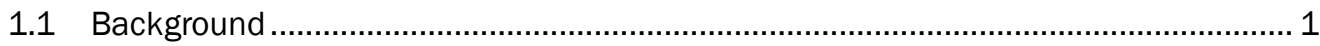

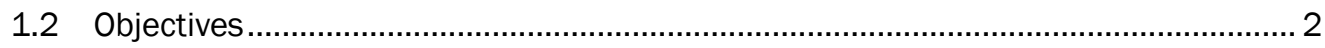

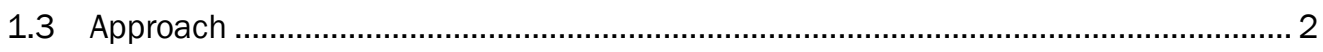

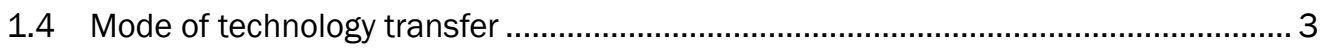

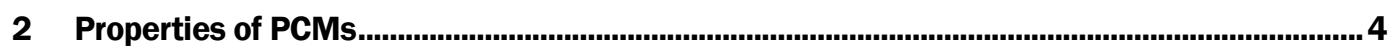

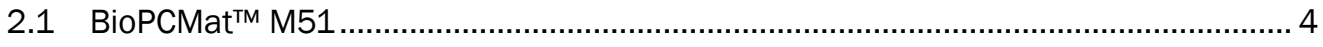

2.2 DuPont ${ }^{\mathrm{TM}}$ Energain $\AA$ Thermal mass panel............................................................... 4

2.3 Micro-encapsulated Phase Change Material (MPCM) ............................................. 5

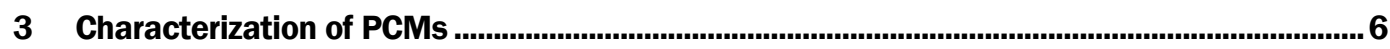

3.1 Sample Preparation ........................................................................................ 6

3.2 Thermal cycling chambers and thermal cycling profile ......................................... 6

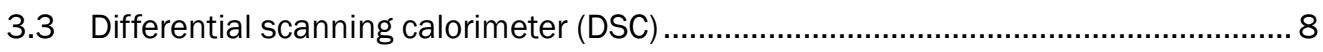

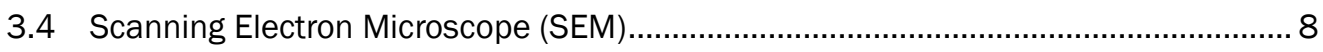

3.5 Fourier transform infrared spectroscopy (FTIR) ............................................ 8

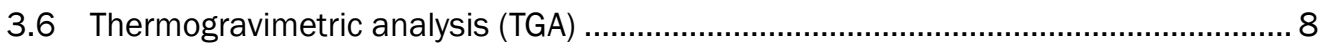

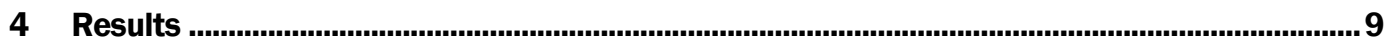

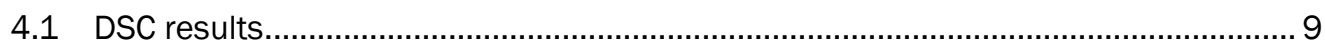

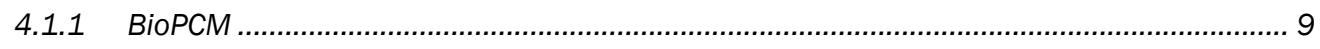

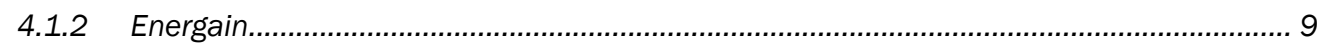

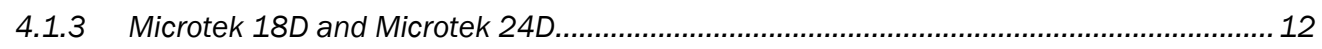

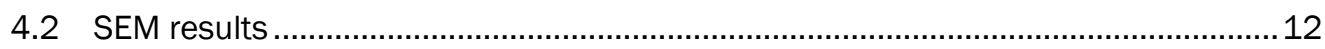

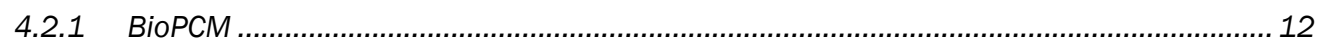

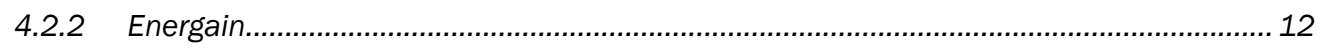

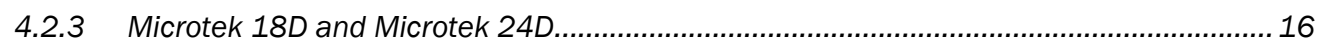

4.3 Fourier Transform Infrared Spectroscopy (FTIR) results ....................................... 17

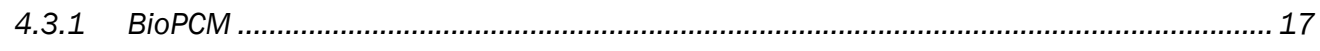

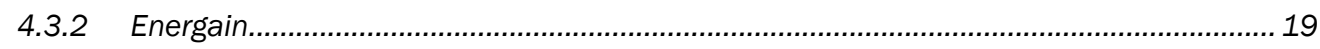

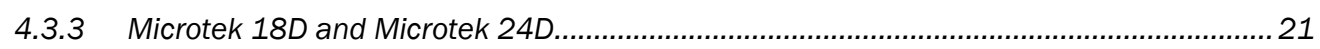

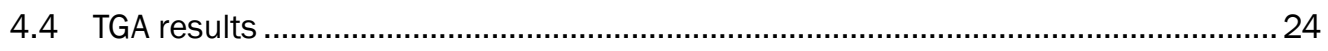

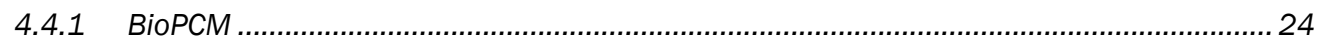

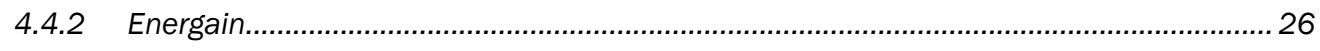

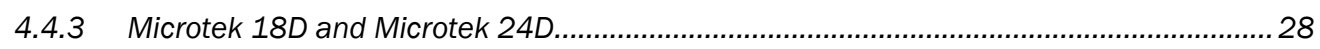

5 Conclusion and Recommendations ....................................................................................31 


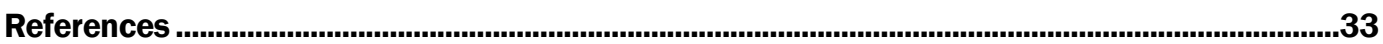

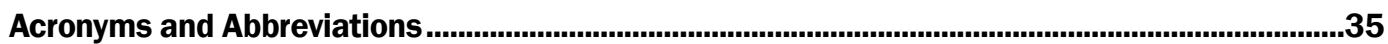

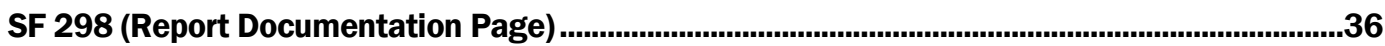




\section{Illustrations}

\section{Figures}

1-1 Classification of PCMs

2-1 BioPCMat ${ }^{\mathrm{TM}}$ materials: (a) blanket with pouches of mixture of palm oil and soy oil, (b) sample put into the environmental chamber for testing, and (c) the appearance of BioPCM sample (extracted from one pouch)

2-2 DuPont ${ }^{\mathrm{TM}}$ Engergain ${ }^{\circledR}$ Thermal mass panel (a) side view of panel, and (b) paraffin wax with elastomer binder sandwiched in between aluminum panels 5

2-3 Micro-encapsulated PCM materials: (a) Microtek 18D sample, and (b) Microtek 24D sample

3-1 Thermal cycling: (a) Sun Electronic Systems EC127 chamber, and (b) thermal cycling profile used for the thermal cycling experiments .7

4-1 Latent heat versus number of cycles for BioPCM M51 …….................................................10

4-2 Latent heat versus number of cycles for Energain

4-3 Latent heat versus number of cycles for micro-encapsulated octadecane mixtures: (a) Microtek $18 \mathrm{D}$ with low melting point $\left(18^{\circ} \mathrm{C}\right)$, (b) Microtek $24 \mathrm{D}$ with high melting point $\left(24^{\circ} \mathrm{C}\right)$.

4-4 SEM images of Micro-encapsulated PCM pellets (Microtek 18D): (a) before thermal cycling,, (b) after 1680 cycling (6.2 years), (c) after 3696 cycling (13.7 years), and (d) after 5400 cycling (20 years)

4-5 SEM images of Micro-encapsulated PCM pellets (Microtek 24D): (a) before thermal cycling,, (b) after 1680 cycling (6.2 years), (c) after 3696 cycling (13.7 years), and (d) after 5400 cycling (20 years)

4-6 SEM images of BioPCM M51: (a) before thermal cycling, (b) after 1680 cycling (6.2 years), (c) after 3696 cycling (13.7 years), and (d) after 5400 cycling (20 years)

4-7 SEM images of Energain: (a) before thermal cycling, and (b) after 5400 cycling (20 years).

4-8 FTIR spectra of BioPCM M51: (a) comparison among four cycles, and (b) comparison between 0 cycle and 5400 cycles

4-9 FTIR spectra of Energain: (a) comparison among four cycles, and (b) comparison between 0 cycle and 5400 cycles.

4-10 FTIR spectra of Microtek 18D: (a) among four cycles, and (b) between 0 cycle and 5400 cycles

4-11 FTIR spectra of Microtek 24D: (a) among four cycles, and (b) between 0 cycle

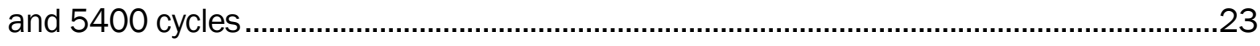

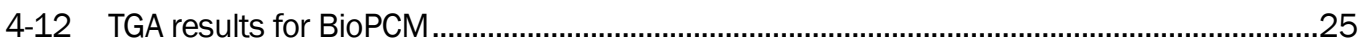

4-13 TGA analysis for Energain PCM................................................................................2

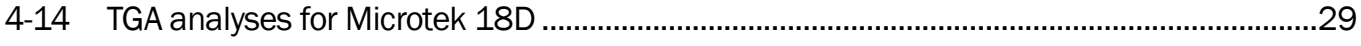

4-15 TGA analyses for Microtek 18D and Microtek 24D.............................................................30 


\section{Tables}

4-1 Differential scanning calorimetry data (melting and freezing) for BioPCM M51 .................10

4-2 Differential scanning calorimetry data (melting and freezing) for Energain ........................11

4-3 Differential scanning calorimetry data (melting and freezing) for Microtek 18D.

Micro-encapsulated PCM with low melting point $\left(18^{\circ} \mathrm{C}\right)$....................................................14

4-4 Differential scanning calorimetry data (melting and freezing) for Microtek 24D, micro-encapsulated PCM with high melting point $\left(24^{\circ} \mathrm{C}\right)$..................................................14

4-5 FTIR spectrum peaks for BioPCM M51 …….............................................................19

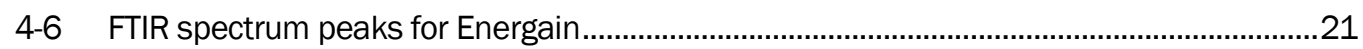

4-7 FTIR spectrum peaks for Microtek 18D and 24D ..............................................................24 


\section{Preface}

This study was conducted for Headquarters, U.S. Army Corps of Engineers (HQUSACE) and the Office of the Assistant Secretary of the Army for Acquisition, Logistics, and Technology, ASA (ALT) under Program Element 622784T45, "Energy Technology Applied to Military Facilities," Project 4451115, "Modeling and Materials for Installations," Task A1180, "Energy Losses in Building Envelopes." This research was also funded by Operational Energy Plans and Programs Office, Office Secretary of Defense under Operational Energy Capital Improvement Fund (OECIF) Program "Advanced, Energy Efficient Shelter Systems for Contingency Basing and Other Applications." The project title was "Advanced Materials for Energy Efficient Shelters." The Program Manager for this ERDC Project was Dr. Ashok Kumar. The technical reviewer was Kurt Kinnevan, CEERD-CV-T.

The work was performed by the Materials and Structures Branch (CF-M) of the Infrastructure Division (CF), U.S. Army Engineer Research and Development Center, Construction Engineering Research Laboratory (ERDCCERL). At the time of publication, Vicki L. Van Blaricum was Chief, CEERD-CF-M; L. Donald K. Hicks was Chief, CEERD-CF; and Kurt Kinnevan, CEERD-CV-T, was the Technical Director for Adaptive and Resilient Installations. The Deputy Director of ERDC-CERL was Dr. Kirankumar Topudurti and the Director was Dr. Ilker Adiguzel.

COLJ effrey R. Eckstein was the Commander of ERDC, and Dr. J effery P. Holland was the Director. 


\section{Introduction}

\subsection{Background}

Phase change materials (PCMs) can absorb or release heat when they undergo phase changes from solid to liquid, liquid to solid, or solid to gas (Royon et al. 1997, Shi et al. 2014). When a PCM solidifies, it releases a large amount of energy in the form of latent heat at a relatively constant temperature. Conversely, when a PCM melts, it absorbs a large amount of heat from the environment. PCMs exhibit a thermal mass effect and repeat these phase changes as ambient temperatures fluctuate. There are many PCMs available, with phase change temperatures that cover a wide range of applications. Figure 1-1 shows a classification of PCMs (Buddhi 2007).

This phase change behavior makes PCMs ideal for use in a variety of everyday applications in certain environments that require temperature control. Over the past decades, for example, PCMs have come to be seen as useful media for used in improving the efficiency of thermal storage systems for application in building envelopes (Castellon et al. 2007, Zhao et al. 2011). Systems that use PCMs have remarkably improved building energy performance (Kosny et al. 2009, Farid et al. 2003). Latent heat storage systems that use PCMs are preferred due to their large energy storage density and practically isothermal nature of the storage process (Sharma et al. 2007, Günther et al. 2006). Note that PCMs are recommended for use in hot, dry, and marine climates with dynamic daytime/ nighttime temperature changes; they are not recommended for use in cold, humid climates.

PCMs are considered especially promising candidates for use in heating and cooling applications for building envelopes because the market for cooling applications is expanding rapidly, and because free cooling systems in existing buildings can be retrofitted. However, the design of a thermal storage system that can serve the narrow temperature range that characterizes a building envelope must be based on reliable and high resolution material data. It is crucial that such a design be based on an accurate determination of the PCM's heat storage capability as a function of temperature. 
Figure 1-1. Classification of PCMs.

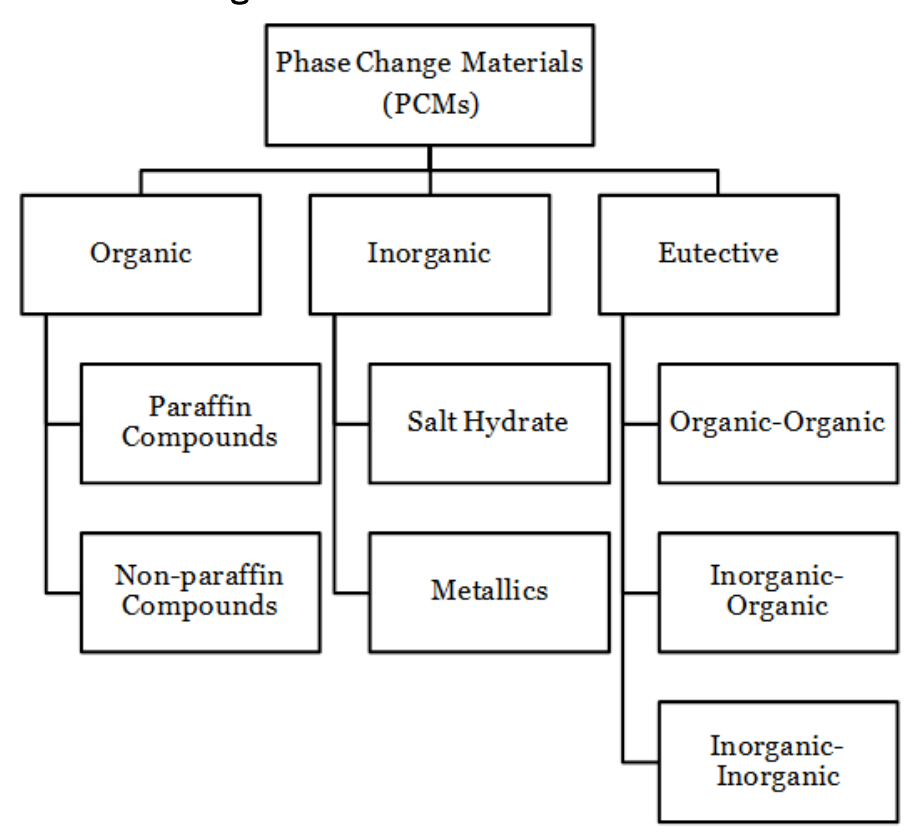

Another very important factor in the design of free cooling applications is the heat transfer between storage material and ambient air. Recent research has focused on simulation and modeling of the PCM based materials, but has not extensively studied the effect of thermal cycling or the stability of the PCMs over extended periods of time. There is a need for experimental measurement of the stability of PCMs.

\subsection{Objectives}

The objectives of this work were to investigate the stability of four commercially available PCMs, and to develop an accelerated testing protocol to simulate the long-term performance of PCMs in an operational scenario for buildings and shelters.

\subsection{Approach}

This work developed an accelerated testing protocol to simulate the longterm performance of PCMs in an operational scenario as follows:

1. The selected PCM s were subjected to up to 5,400 cycles of thermal cycling at 90 minutes per cycle, over the temperature range of $14-140{ }^{\circ} \mathrm{F}(-10$ $60{ }^{\circ} \mathrm{C}$ ), to simulate 20 years of use in building envelopes.

2. At 3-4 week time intervals, the samples were taken from the thermal cycling chamber and Scanning Electron Microscope (SEM) micrographs, and Differential scanning calorimeter (DSC), TGA, and FTIR data were obtained and compared to baseline (pre-cycling data). 
3. Baseline latent heat values and freezing and melting transition temperatures were determined.

4. These gathered data were used to evaluate the stability of PCMs based on the differences in latent heat storage and to predict thelong-term performance of four selected PCMs and their future application.

\subsection{Mode of technology transfer}

This report will be made accessible through the World Wide Web at URL: http://libweb.erdc.usace.army.mil 


\section{Properties of PCMs}

Four commercially available PCMs, which represent different chemical natures, were selected for testing: (1) BioPCMat ${ }^{\mathrm{TM}}$ M51 (PHASECHANGE Energy Solution, Asheboro, NC), (2) DuPont ${ }^{\mathrm{TM}}$ Energain ${ }^{\circledR}$ Thermal mass panel (DuPont, Wilmington, DE), and (3, 4) Micro-encapsulated Phase Change Material (MPCM) with two different melting and freezing points (Microtek, Laboratories, Inc., Dayton, OH). The following sections describe each material.

\section{$2.1 \quad$ BioPCMat $^{\mathrm{TM}}$ M51}

In practice, BioPCMat ${ }^{\mathrm{TM}}$ materials are used as the core materials of a PCM wall or ceiling panel. BioPCMat ${ }^{\mathrm{TM}}$ absorbs heat in the daytime and releases that heat during the night. The dimension of the typical blanket is either $48 \times 16.5$ in. (1.22 x $0.42 \mathrm{~m}$ ) or 96 x $16.5 \mathrm{in}$. (2.44 $0.42 \mathrm{~m}$ ) (Figure 2-1). This material also contains fire retardant material. This research extracted BioPCM samples from one of the polymeric pouches of a blanket and subjected it to thermal cycling.

Figure 2-1. BioPCMat ${ }^{\mathrm{TM}}$ materials: (a) blanket with pouches of mixture of palm oil and soy oil, (b) sample put into the environmental chamber for testing, and (c) the appearance of BioPCM sample (extracted from one pouch).

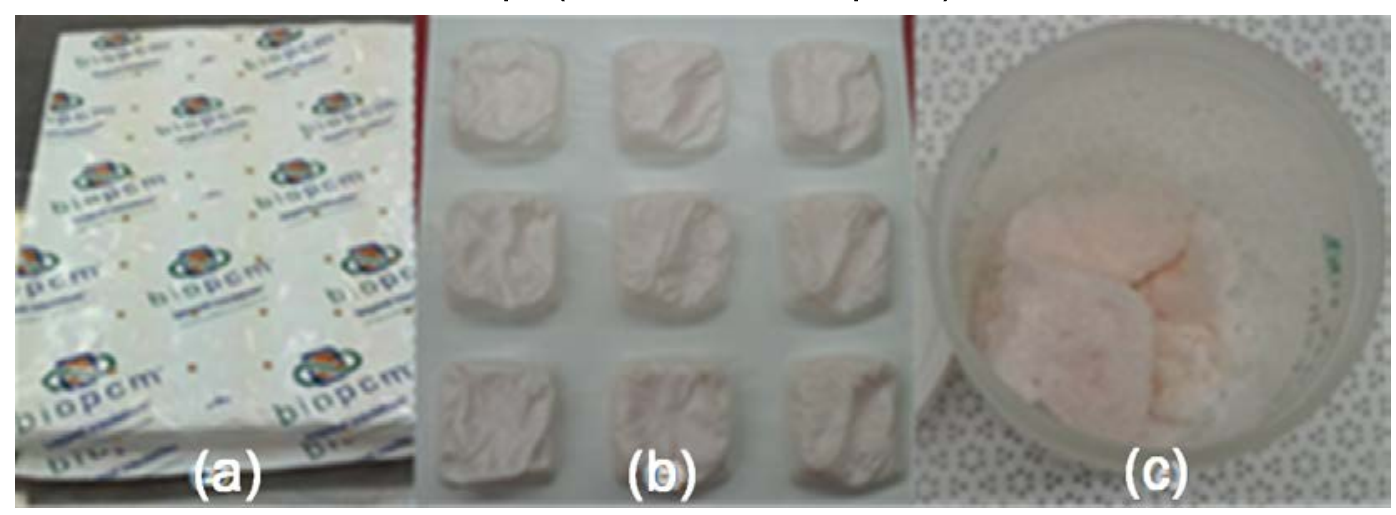

\subsection{DuPont ${ }^{\mathrm{TM}}$ Energain ${ }^{\circledR}$ Thermal mass panel}

The DuPont ${ }^{\mathrm{TM}}$ Engergain ${ }^{\circledR}$ Thermal mass panel (Figure 2-2) is a fine mixture of ethylene based polymer (40\%) and paraffin wax (60\%) sandwiched between two thin aluminum sheets. According to the manufacturer's information, DuPont ${ }^{\mathrm{TM}}$ Energain ${ }^{\circledR}$ significantly decreases air-conditioning costs by an average of $35 \%$ and heating costs by about $15 \%$, and can contribute considerably to the reduction a building's carbon footprint. The system 
consists of lightweight, easy-to-install panels of 3.28 x $3.94 \mathrm{ft}, 0.21 \mathrm{in}$. thick ( $1 \times 1.2 \mathrm{~m}, 5.26 \mathrm{~mm}$ thick). This material also contains an elastomer binder to hold the paraffin in place to prevent sagging and dripping as it melts. This research extracted Energain samples from one of the panels and subjected it to thermal cycling.

Figure 2-2. DuPont ${ }^{\mathrm{TM}}$ Engergain ${ }^{\circledR}$ Thermal mass panel (a) side view of panel, and (b) paraffin wax with elastomer binder sandwiched in between aluminum panels.

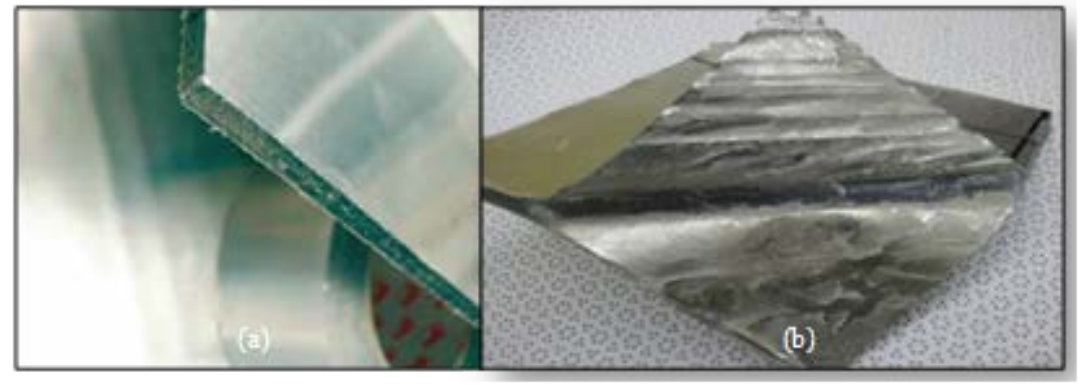

\subsection{Micro-encapsulated Phase Change Material (MPCM)}

Two commercially available micro-encapsulated PCM materials with different melting and freezing points (Microtek 18D and Microtek 24D, respectively) were selected. According to the manufacturer's data, micro-encapsulated PCM has excellent chemical and thermal stability and can be directly incorporated into insulation materials. The appearances of both materials are white to slightly off-white color in a dry powder form. The core material is octadecane mixtures with mean particle size around 17$20 \mu \mathrm{m}$ (0.67-0.79 mil). Due to its powdery consistency, this material can be directly incorporated into cellulose insulation materials.

Figure 2-3. Micro-encapsulated PCM materials: (a) Microtek 18D sample, and (b) Microtek 24D sample.

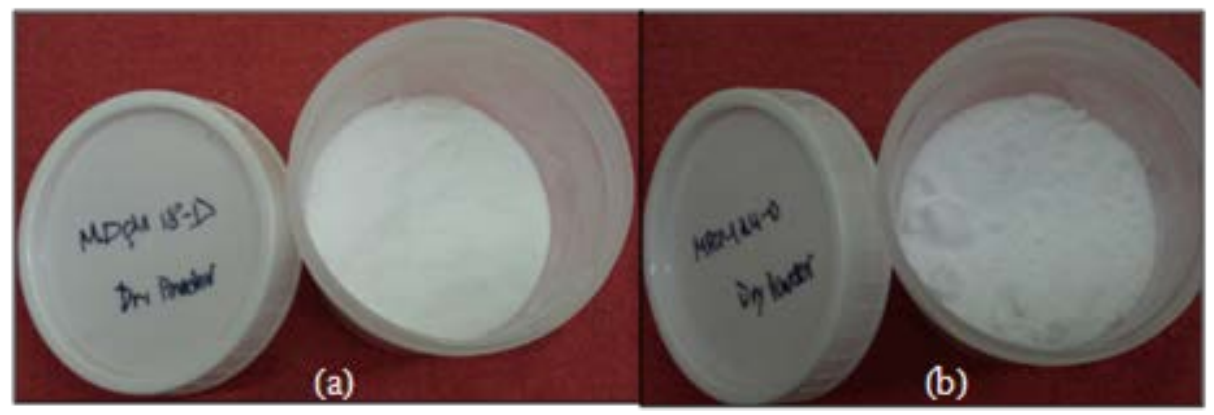




\section{Characterization of PCMs}

\subsection{Sample Preparation}

Samples of two PCMs including Microtek 18D and Microtek 24D were placed into two glass vials. One 3 x 3-in. (76.2 x $76.2 \mathrm{~mm}$ ) pouch of BioPCM was cut off from its original polymer blanket. The manufacturer provided a 1-in. (25.4 mm) Energain panel. All these four samples were placed into the cycling chamber. Baseline DSC, SEM, FTIR, and TGA measurements were taken for each sample before thermal cycling. At 3week intervals, each sample was removed from the thermal cycling chamber. An adequate quantity of material was taken from each sample and DSC, SEM, FTIR, and TGA measurements were taken to determine any changes in their physical, chemical, and thermal properties. The remaining samples were returned to the thermal cycling chamber to continue the long-term, 24/ 7 cycling test.

\subsection{Thermal cycling chambers and thermal cycling profile}

Cycling test was performed in Sun Electronic Systems, Inc. (Titusville, FL) Model EC127 temperature test chambers (Figure 3-1a). The thermal cycling was conducted under controlled temperature conditions. A small battery-operated thermometer within the thermal cycling chamber provided independent verification of the chamber temperatures. Thermal cycling began at $14^{\circ} \mathrm{F}$ $\left(-10^{\circ} \mathrm{C}\right)$ and was ramped up to $140^{\circ} \mathrm{F}\left(60^{\circ} \mathrm{C}\right)$ with heating rate of $38.3^{\circ} \mathrm{Fmin}^{-1}\left(3.5^{\circ} \mathrm{Cmin}^{-1}\right)$. The samples were held at $140^{\circ} \mathrm{F}\left(60^{\circ} \mathrm{C}\right)$ for 25 minutes, and were subsequently cooled to $14^{\circ} \mathrm{F}\left(-10^{\circ} \mathrm{C}\right)$ at a cooling rate of $38.3^{\circ} \mathrm{Fmin}^{-1}\left(3.5^{\circ} \mathrm{C} \mathrm{min}^{-1}\right)$. Each complete cycle, which represents one full solid-to-liquid-to-solid cycle, requires 90 minutes.

It was assumed that 272 complete cycles ( 17 days with duration of 90 minutes for each cycle) simulated 1 year of use (Gao et al. 2013). This assumption is based on the observation that, in most building applications, temperatures will either be excessively hot or excessively cold at certain times of the year. As a result, the $\mathrm{PCM}$ will not undergo phase changes during one-quarter of theyear. Therefore, over a one year period, the simulation subjected these four materials to 5,400 thermal cycles, which simulated 20 years of use in building envelopes. 
Figure 3-1. Thermal cycling: (a) Sun Electronic Systems EC127 chamber, and

(b) thermal cycling profile used for the thermal cycling experiments.
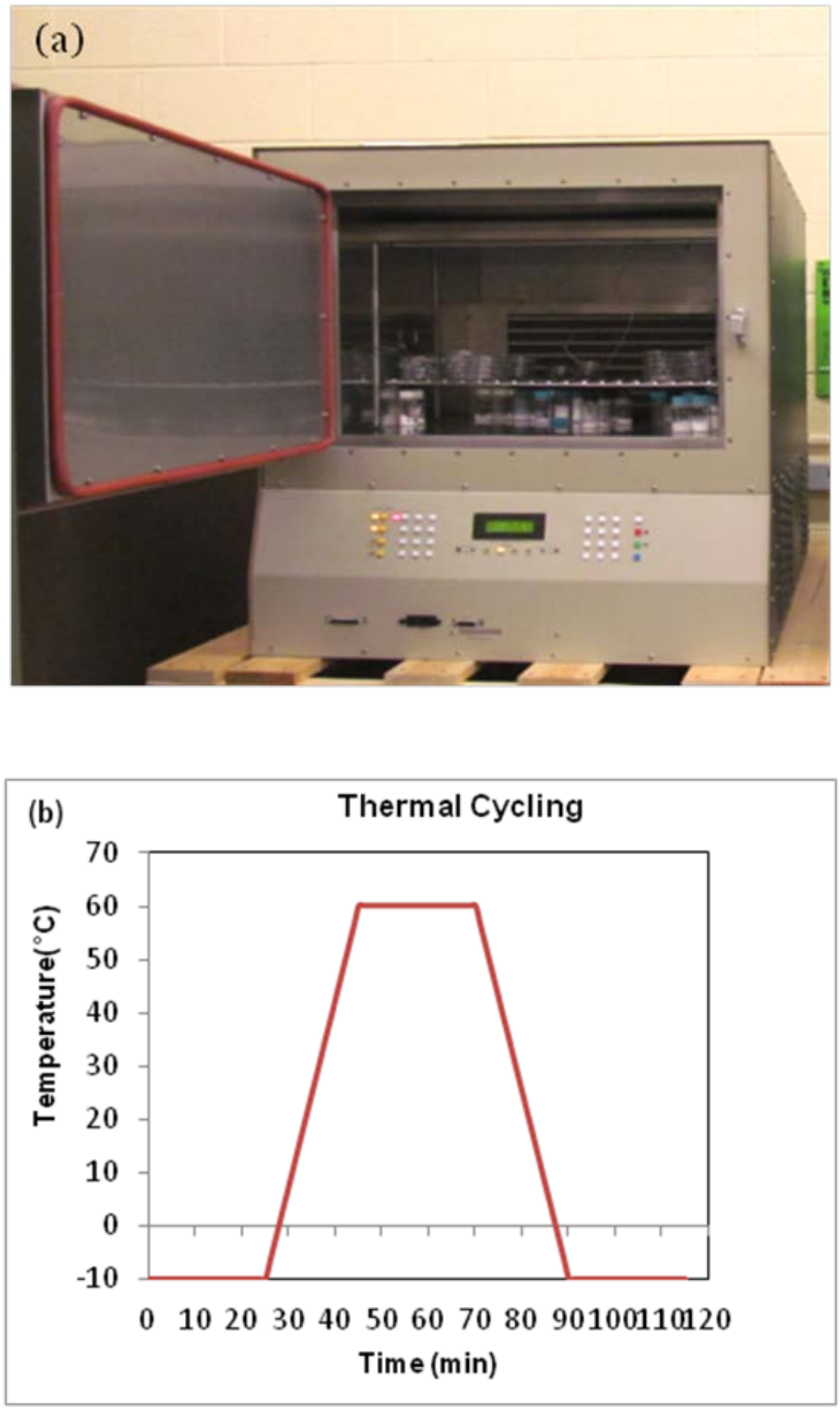


\subsection{Differential scanning calorimeter (DSC)}

A TA Instrument Model Q20 DSC (New Castle, DE) was used to analyze PCM thermal properties per American Society for Testing and Materials (ASTM) E 1269 (2011), including melting and freezing points, heat of fusion, melting and freezing onset, and peak transition range. Aluminum sealed aluminum pans, purchased from TA instrument, Inc. were used for sample preparation. Nitrogen gas at a flow rate of $50 \mathrm{ml}^{3} \mathrm{~min}^{-1}$ was used as purge gas. To minimize the variation in DSC measurements, all samples weighed approximately between 0.00018 and $0.00021 \mathrm{oz}$ ( 5 and $6 \mathrm{mg}$ ), and three DSC pans were prepared for each PCM. Each DSC measurement was conducted at constant heating and cooling rate of $33.8^{\circ} \mathrm{Fmin}^{-1}$ $\left(1^{\circ} \mathrm{Cmin}^{-1}\right)$ and repeated three times for each sample. The beginning and ending temperatures of the DSC scans for both PCM were $14^{\circ} \mathrm{F}\left(-10^{\circ} \mathrm{C}\right)$ and $104^{\circ} \mathrm{F}\left(40^{\circ} \mathrm{C}\right)$, respectively.

\subsection{Scanning Electron Microscope (SEM)}

A model USM-6390 SEM was purchased from JEOL (Peabody, MA). SEM was used to characterize the morphology, size, and any thermal-cycling-induced micro-structure changes in the samples. Conductive carbon adhesive tape was attached to a SEM stub and the sample was dispersed on the tape. Gold-coating was sputtered using model Desk II (Denton Vacuum, Moorestown, NJ). The size of microcapsules was measured on the SEM photos.

\subsection{Fourier transform infrared spectroscopy (FTIR)}

To determine the quality or consistency of selected PCMs, FTIR spectroscopy was conducted using a J ASCO FTIR-4100 (Easton, MD). A small amount of sample was dispersed onto an attenuated total reflectance (ATR) detector to fill dwell. The scan range was between 400 and $4000 \mathrm{~cm}^{-1}$. The FTIR spectrograph was further corrected by means of the inbuilt analysis tool for presentation purpose. The infrared (IR) spectra for each cycle interval were compared with the baseline (without cycling) spectrum

\subsection{Thermogravimetric analysis (TGA)}

The thermal stability of PCM was measured using a Shimadzu Model TGA50 thermogravimetric analyzer (Tokyo, J apan) with nitrogen as a purge gas at a flow rate of $6.1 \mathrm{cu}$ in. $\mathrm{min}^{-1}\left(100 \mathrm{ml}^{3} \mathrm{~min}-1\right)$. All the samples weighed between 4 and $9 \mathrm{mg}(0.00014$ and $0.00032 \mathrm{oz}$.) and were heated from ambient to $500^{\circ} \mathrm{C}\left(932^{\circ} \mathrm{F}\right)$. 


\section{Results}

\subsection{DSC results}

\subsubsection{BioPCM}

Thermal analysis of the BioPCM M51 was measured by DSC. Figure 4-1 shows the changes in latent heat in the baseline and after 1680, 3696, and 5376 thermal cycles; the heating and freezing curves are for the baseline and each cycle on the DSC, respectively. Table 4-1 lists the DSC results in terms of melting point, freezing point, and the latent heat for the baseline and for each set of cycles. The latent heat of the BioPCM M51 changed $11.82 \%$ and $11.72 \%$ from the baseline after 1,680 cycles for melting and freezing, respectively. After 1,680 cycles, the latent heat changed by $1 \%$ and $3.8 \%$ for melting and freezing, respectively. However, the melting point temperature remained in range of 79.8 to $80.8{ }^{\circ} \mathrm{F}$ (26.56 to $27.12{ }^{\circ} \mathrm{C}$ ) and freezing point in range of 70 to $-7.744^{\circ} \mathrm{F}\left(21.10\right.$ to $\left.-22.08{ }^{\circ} \mathrm{C}\right)$ for the baseline and each thermal cycle. The results indicate the BioPCM M51 has stable melting and freezing points. The latent heat tended to stabilize over the later cycles in the range of 0.02773 to $0.02801 \mathrm{Btu} /(\mathrm{lb} . \mathrm{F})$ (116.10 to $117.27 \mathrm{~J} / \mathrm{g})$ for melting and 0.02679 to $0.02802 \mathrm{Btu} / \mathrm{lb} . \mathrm{F})(112.17 \mathrm{~J} / \mathrm{g}$ to $117.3 \mathrm{~J} / \mathrm{g}$ ) for freezing.

\subsubsection{Energain}

DSC indicated that, for Energain, the onset of the transition temperatures for the freezing curve changed from 67 to $80^{\circ} \mathrm{F}$ ( 19.4 to $26.55^{\circ} \mathrm{C}$ ) after 1,680 cycles, while the melting curve shifted from 61 to $64^{\circ} \mathrm{F}(15.9$ t0 $\left.17.5^{\circ} \mathrm{C}\right)$, with a concomitant loss of latent heat ( 12-14\%) during the both melting and freezing cycles (Figure 4-2 and Table 4-2). After 3,696 cycles, these values show significant recovery toward their pre-cycled (baseline) values. At the end of the 5400-cycle simulation, transition temperature for the freezing curve had recovered the pre-cycled values, while the temperature onset for the melting curve showed a net increase by $2^{\circ} \mathrm{F}\left(1.1^{\circ} \mathrm{C}\right)$. Further cycling shows recovery of pre-cycled latent heat values, and even a slight increase $(\sim 0.00096 \mathrm{Btu} /(\mathrm{lb} . \mathrm{F})[\sim 4 \mathrm{~J} / \mathrm{g}])$. 
Figure 4-1. Latent heat versus number of cycles for BioPCM M51.

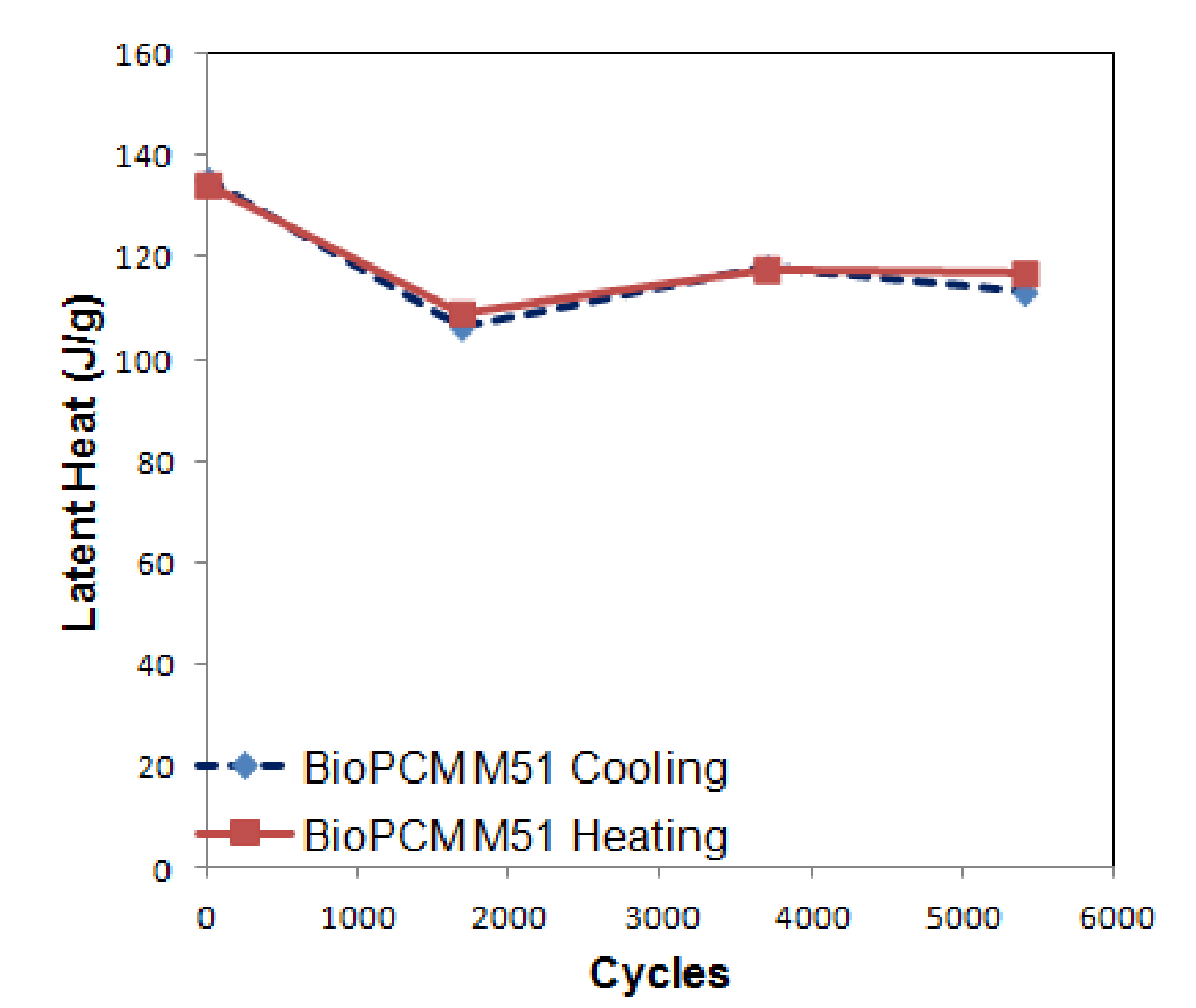

Table 4-1. Differential scanning calorimetry data (melting and freezing) for BioPCM M51.

\begin{tabular}{|c|c|c|c|c|c|c|c|}
\hline \multicolumn{8}{|c|}{ Melting } \\
\hline \multirow[b]{2}{*}{ Cycles } & \multirow[b]{2}{*}{ Weeks } & \multicolumn{2}{|c|}{ Onset Average } & \multicolumn{2}{|c|}{ Melting Point Average } & \multicolumn{2}{|c|}{ Latent Heat Average } \\
\hline & & ${ }^{\circ} \mathrm{F}$ & ${ }^{\circ} \mathrm{C}$ & ${ }^{\circ} \mathrm{F}$ & ${ }^{\circ} \mathrm{C}$ & Btu/(Ib.F) & $\mathrm{J} / \mathrm{g}$ \\
\hline 0 & 0 & 72.9 & 22.74 & 79.8 & 26.56 & 0.032 & 133.00 \\
\hline 1680 & 15 & 74.2 & 23.46 & 80.6 & 27.00 & 0.028 & 117.27 \\
\hline 3696 & 33 & 75.1 & 23.93 & 80.1 & 26.72 & 0.028 & 117.80 \\
\hline 5400 & 48 & 75.0 & 23.90 & 80.8 & 27.12 & 0.028 & 116.10 \\
\hline \multicolumn{8}{|c|}{ Freezing } \\
\hline \multirow[t]{2}{*}{ Cycles } & \multirow[t]{2}{*}{ Weeks } & \multicolumn{2}{|c|}{ Onset Average } & \multicolumn{2}{|c|}{ Freezing Point Average } & \multicolumn{2}{|c|}{ Latent Heat Average } \\
\hline & & ${ }^{\circ} \mathrm{F}$ & ${ }^{\circ} \mathrm{C}$ & ${ }^{\circ} \mathrm{F}$ & ${ }^{\circ} \mathrm{C}$ & Btu/(Ib.F) & $\mathrm{J} / \mathrm{g}$ \\
\hline 0 & 0 & 71.8 & 22.09 & 70.0 & 21.10 & 0.032 & 132.90 \\
\hline 1680 & 15 & 74.5 & 23.62 & 71.2 & 21.77 & 0.028 & 117.30 \\
\hline 3696 & 33 & 73.2 & 22.90 & 71.1 & 21.70 & 0.029 & 119.53 \\
\hline 5400 & 48 & 74.9 & 23.85 & 71.7 & 22.08 & 0.027 & 112.17 \\
\hline
\end{tabular}


Figure 4-2. Latent heat versus number of cycles for Energain.

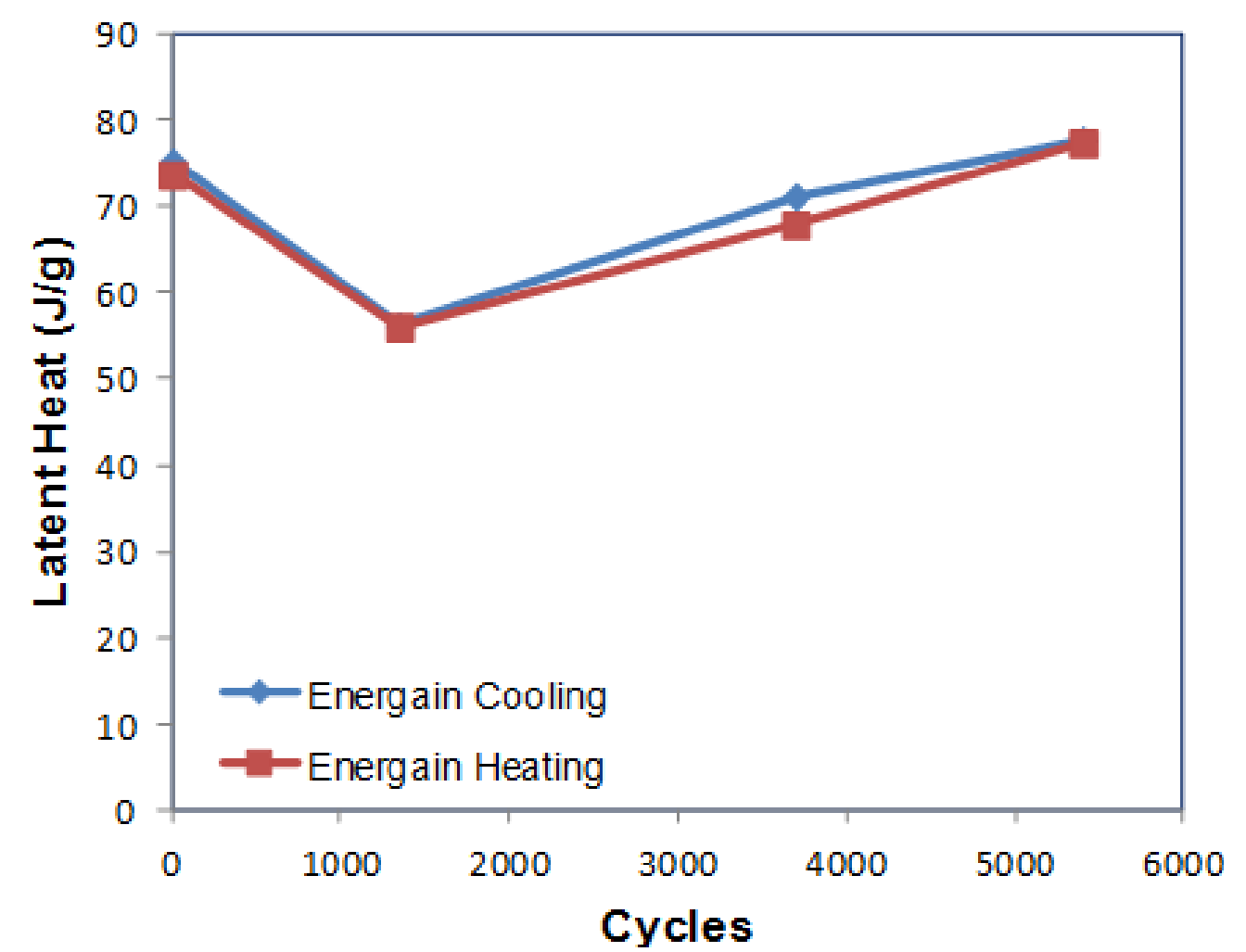

Table 4-2. Differential scanning calorimetry data (melting and freezing) for Energain.

\begin{tabular}{|c|c|c|c|c|c|c|c|}
\hline \multicolumn{8}{|c|}{ Melting } \\
\hline \multirow[b]{2}{*}{ Cycles } & \multirow[b]{2}{*}{ Weeks } & \multicolumn{2}{|c|}{ Onset Average } & \multicolumn{2}{|c|}{ Melting Point Average } & \multicolumn{2}{|c|}{ Latent Heat Average } \\
\hline & & ${ }^{\circ} \mathrm{F}$ & ${ }^{\circ} \mathrm{C}$ & ${ }^{\circ} \mathrm{F}$ & ${ }^{\circ} \mathrm{C}$ & Btu/(lb.F) & $\mathrm{J} / \mathrm{g}$ \\
\hline 0 & 0 & 60.71 & 15.95 & 72.14 & 22.30 & 0.018 & 73.58 \\
\hline 1680 & 15 & 63.45 & 17.47 & 74.64 & 23.69 & 0.015 & 61.36 \\
\hline 3696 & 33 & 62.22 & 16.79 & 71.15 & 21.75 & 0.016 & 67.86 \\
\hline 5400 & 48 & 64.26 & 17.92 & 72.59 & 22.55 & 0.018 & 77.39 \\
\hline \multicolumn{8}{|c|}{ Freezing } \\
\hline \multirow[b]{2}{*}{ Cycles } & \multirow[b]{2}{*}{ Weeks } & \multicolumn{2}{|c|}{ Onset Average } & \multicolumn{2}{|c|}{ Freezing Point Average } & \multicolumn{2}{|c|}{ Latent Heat Average } \\
\hline & & ${ }^{\circ} \mathrm{F}$ & ${ }^{\circ} \mathrm{C}$ & ${ }^{\circ} \mathrm{F}$ & ${ }^{\circ} \mathrm{C}$ & Btu/(Ib.F) & $\mathrm{J} / \mathrm{g}$ \\
\hline 0 & 0 & 66.94 & 19.41 & 62.67 & 17.04 & 0.018 & 75.41 \\
\hline 1680 & 15 & 79.79 & 26.55 & 71.89 & 22.16 & 0.015 & 61.37 \\
\hline 3696 & 33 & 70.56 & 21.42 & 59.99 & 15.55 & 0.017 & 71.19 \\
\hline 5400 & 48 & 66.74 & 19.30 & 63.01 & 17.23 & 0.019 & 77.72 \\
\hline
\end{tabular}




\subsubsection{Microtek 18D and Microtek 24D}

Figure 4-3 shows the DSC curve of Microtek 18D and Microtek 24D, which indicate that the melting point of Microtek 18D was depressed up to $39.2^{\circ} \mathrm{F}\left(4^{\circ} \mathrm{C}\right)$. The melting point of Microtek 24D changed very little. The DSC curves Figure 4-3 may be used to compare the pre-cycled sample with the sample that underwent 5400 cycles. Tables 4-3 and 4-4 summarize the onset temperature, melting point, and latent heat for both samples.

Figure 4-3a and b show the DSC curves of Microtek 18D and Microtek 24D. These results show that the melting point of Microtek 18D was depressed up to $7.2^{\circ} \mathrm{F}\left(4^{\circ} \mathrm{C}\right)$. The latent heat of 18D shown by the heating (melting) curve decreased steadily with increasing thermal cycles, and was severely reduced from 0.0406 to $0.03583 \mathrm{Btu} /(\mathrm{lb} . \mathrm{F})$ (170 to $150 \mathrm{~J} / \mathrm{g})$ after 5,400 cycles. The latent heat of $18 \mathrm{D}$ shown by the cooling (freezing) curve also decreased steadily with increasing thermal cycles, and was severely reduced from 0.0406 to $0.02866 \mathrm{Btu} /(\mathrm{lb} . \mathrm{F})$ (170 to $120 \mathrm{~J} / \mathrm{g}$ ) after 5,400 cycles. The melting point of Microtek 24D showed insignificant change. Tables 4-3 and 4-4 summarize the onset temperature, melting point, and latent heat for both samples.

\subsection{SEM results}

\subsubsection{BioPCM}

SEM micrographs for BioPCM (Figure 4-4) indicated that, after 1680 aging cycles, the BioPCM baseline surface morphology appeared to change from a uniform mix of PCM materials with micro particles to a matrix of scattered micro particles; however, it did not reveal significant damage or impurities.

\subsubsection{Energain}

SEM micrographs (Figure 4-5) showed that, after being subjected to 5,400 thermal cycles, the morphology of Energain PCM samples demonstrated evidence of melting and recrystallization. Randomly distributed large discontinuities (pock marks) in the material disappeared and were replaced by smaller more shallow discontinuities aligned in a preferred direction. In addition, the surface morphology became much smoother than the baseline samples. This observation was consistent with many cycles of melting, cooling, and recrystallization. No significant damage or impurities were observed in the micrographs. 
Figure 4-3. Latent heat versus number of cycles for micro-encapsulated octadecane mixtures: (a) Microtek $18 \mathrm{D}$ with low melting point $\left(18^{\circ} \mathrm{C}\right)$, (b) Microtek $24 \mathrm{D}$ with high melting point $\left(24^{\circ} \mathrm{C}\right)$.
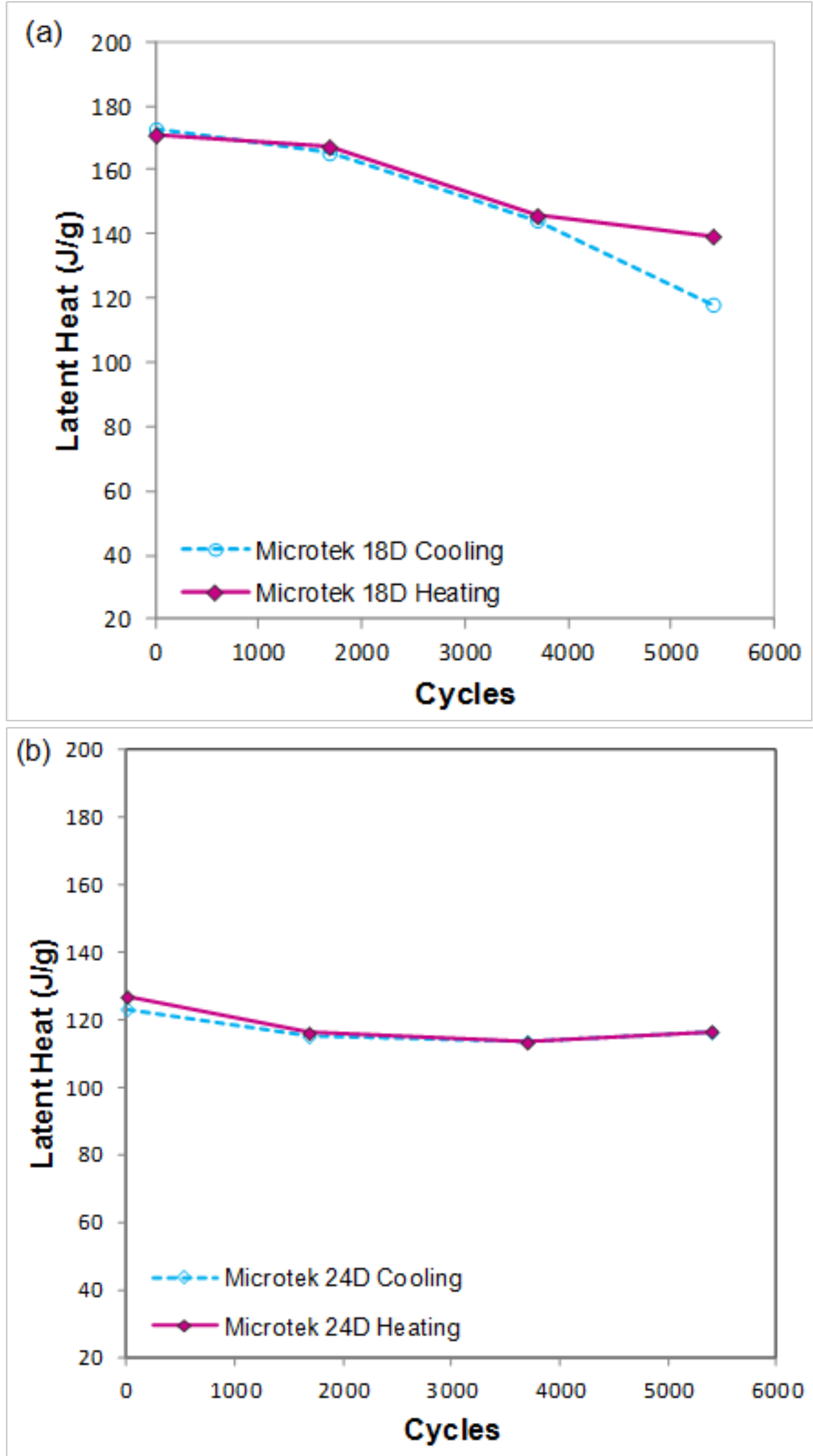
Table 4-3. Differential scanning calorimetry data (melting and freezing) for Microtek 18D. Micro-encapsulated PCM with low melting point $\left(18^{\circ} \mathrm{C}\right)$.

\begin{tabular}{|c|c|c|c|c|c|c|c|}
\hline \multicolumn{8}{|c|}{ Melting } \\
\hline \multirow[b]{2}{*}{ Cycles } & \multirow[b]{2}{*}{ Weeks } & \multicolumn{2}{|c|}{ Onset Average } & \multicolumn{2}{|c|}{ Melting Point Average } & \multicolumn{2}{|c|}{ Latent Heat Average } \\
\hline & & ${ }^{\circ} \mathrm{F}$ & ${ }^{\circ} \mathrm{C}$ & ${ }^{\circ} \mathrm{F}$ & ${ }^{\circ} \mathrm{C}$ & Btu/(Ib.F) & $\mathrm{J} / \mathrm{g}$ \\
\hline 0 & 0 & 57.97 & 14.43 & 63.12 & 17.29 & 0.041 & 170.90 \\
\hline 1680 & 15 & 56.57 & 13.65 & 61.97 & 16.65 & 0.040 & 167.20 \\
\hline 3696 & 33 & 57.49 & 14.16 & 61.70 & 16.50 & 0.035 & 145.74 \\
\hline 5400 & 48 & 51.73 & 10.96 & 58.19 & 14.55 & 0.033 & 139.37 \\
\hline \multicolumn{8}{|c|}{ Freezing } \\
\hline \multirow[t]{2}{*}{ Cycles } & \multirow[t]{2}{*}{ Weeks } & \multicolumn{2}{|c|}{ Onset Average } & \multicolumn{2}{|c|}{ Freezing Point Average } & \multicolumn{2}{|c|}{ Latent Heat Average } \\
\hline & & ${ }^{\circ} \mathrm{F}$ & ${ }^{\circ} \mathrm{C}$ & ${ }^{\circ} \mathrm{F}$ & ${ }^{\circ} \mathrm{C}$ & Btu/(Ib.F) & $\mathrm{J} / \mathrm{g}$ \\
\hline 0 & 0 & 58.21 & 14.56 & 55.45 & 13.03 & 0.041 & 172.72 \\
\hline 1680 & 15 & 58.64 & 14.80 & 57.06 & 13.92 & 0.039 & 165.33 \\
\hline 3696 & 33 & 58.91 & 14.95 & 56.35 & 53 & 0.034 & 144.37 \\
\hline 5400 & 48 & 56.95 & 13.86 & 55.18 & 12.88 & 0.028 & 118.13 \\
\hline
\end{tabular}

Table 4-4. Differential scanning calorimetry data (melting and freezing) for Microtek 24D, micro-encapsulated PCM with high melting point $\left(24^{\circ} \mathrm{C}\right)$.

\begin{tabular}{|c|c|c|c|c|c|c|c|}
\hline \multicolumn{8}{|c|}{ Melting } \\
\hline \multirow[b]{2}{*}{ Cycles } & \multirow[b]{2}{*}{ Weeks } & \multicolumn{2}{|c|}{ Onset Average } & \multicolumn{2}{|c|}{ Melting Point Average } & \multicolumn{2}{|c|}{ Latent Heat Average } \\
\hline & & ${ }^{\circ} \mathrm{F}$ & ${ }^{\circ} \mathrm{C}$ & ${ }^{\circ} \mathrm{F}$ & ${ }^{\circ} \mathrm{C}$ & Btu/(Ib.F) & $\mathrm{J} / \mathrm{g}$ \\
\hline 0 & 0 & 66.43 & 19.13 & 72.37 & 22.43 & 0.030 & 126.70 \\
\hline 1680 & 15 & 66.18 & 18.99 & 72.27 & 22.37 & 0.028 & 115.97 \\
\hline 3696 & 33 & 66.52 & 19.18 & 72.66 & 22.59 & 0.027 & 113.17 \\
\hline 5400 & 48 & 66.29 & 19.05 & 72.27 & 22.37 & 0.028 & 116.43 \\
\hline \multicolumn{8}{|c|}{ Freezing } \\
\hline \multirow[b]{2}{*}{ Cycles } & \multirow[b]{2}{*}{ Weeks } & \multicolumn{2}{|c|}{ Onset Average } & \multicolumn{2}{|c|}{ Freezing Point Average } & \multicolumn{2}{|c|}{ Latent Heat Average } \\
\hline & & ${ }^{\circ} \mathrm{F}$ & ${ }^{\circ} \mathrm{C}$ & ${ }^{\circ} \mathrm{F}$ & ${ }^{\circ} \mathrm{C}$ & Btu/(Ib.F) & $\mathrm{J} / \mathrm{g}$ \\
\hline 0 & 0 & 71.73 & 22.07 & 71.01 & 21.67 & 0.029 & 123.11 \\
\hline 1680 & 15 & 72.14 & 22.30 & 70.47 & 21.37 & 0.027 & 114.98 \\
\hline 3696 & 33 & 71.89 & 22.16 & 70.70 & 21.50 & 0.027 & 113.53 \\
\hline 5400 & 48 & 71.80 & 22.11 & 70.70 & 21.50 & 0.028 & 116.23 \\
\hline
\end{tabular}


Figure 4-4. SEM images of Micro-encapsulated PCM pellets (Microtek 18D): (a) before thermal cycling,, (b) after 1680 cycling (6.2 years), (c) after 3696 cycling (13.7 years), and (d) after 5400 cycling ( 20 years).
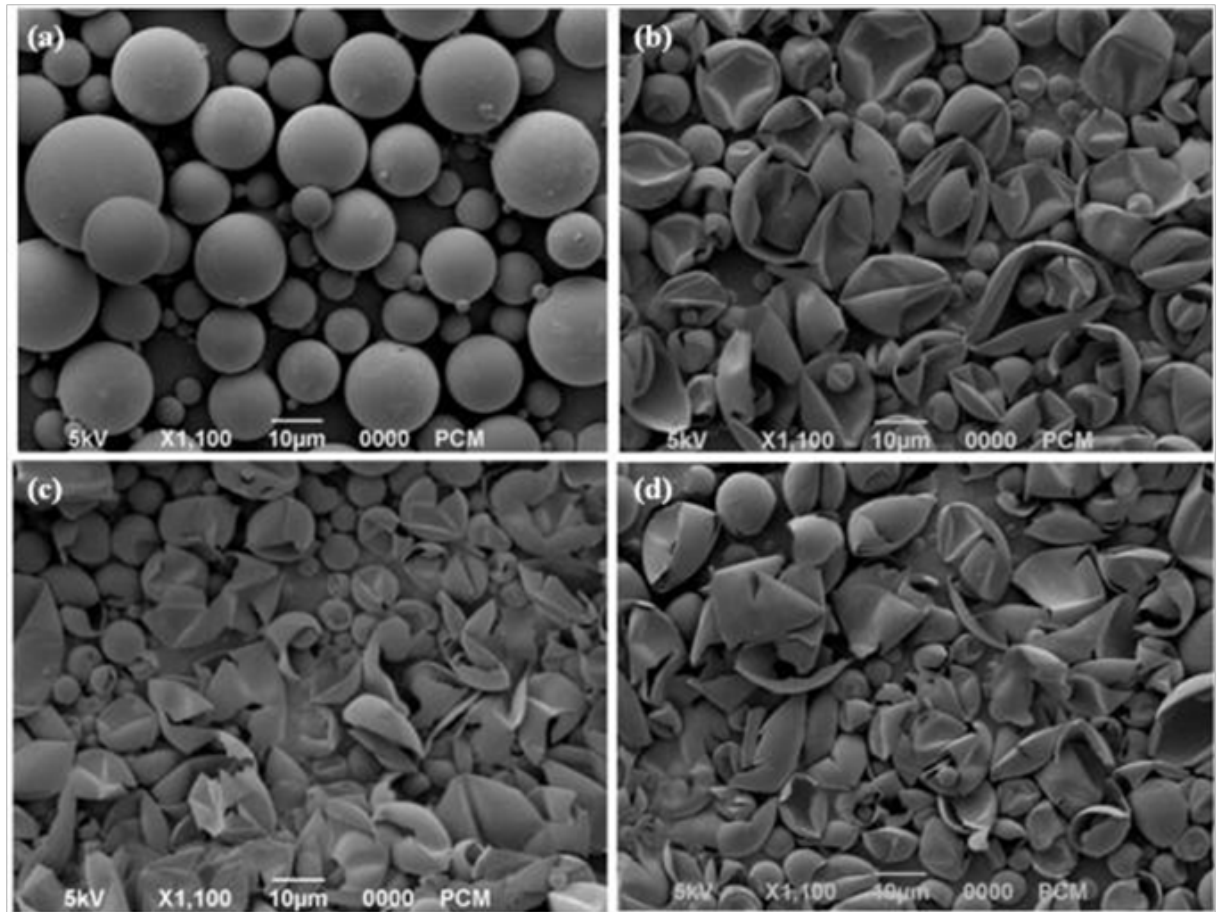

Figure 4-5. SEM images of Micro-encapsulated PCM pellets (Microtek 24D): (a) before thermal cycling,, (b) after 1680 cycling (6.2 years), (c) after 3696 cycling (13.7 years), and

(d) after 5400 cycling (20 years).
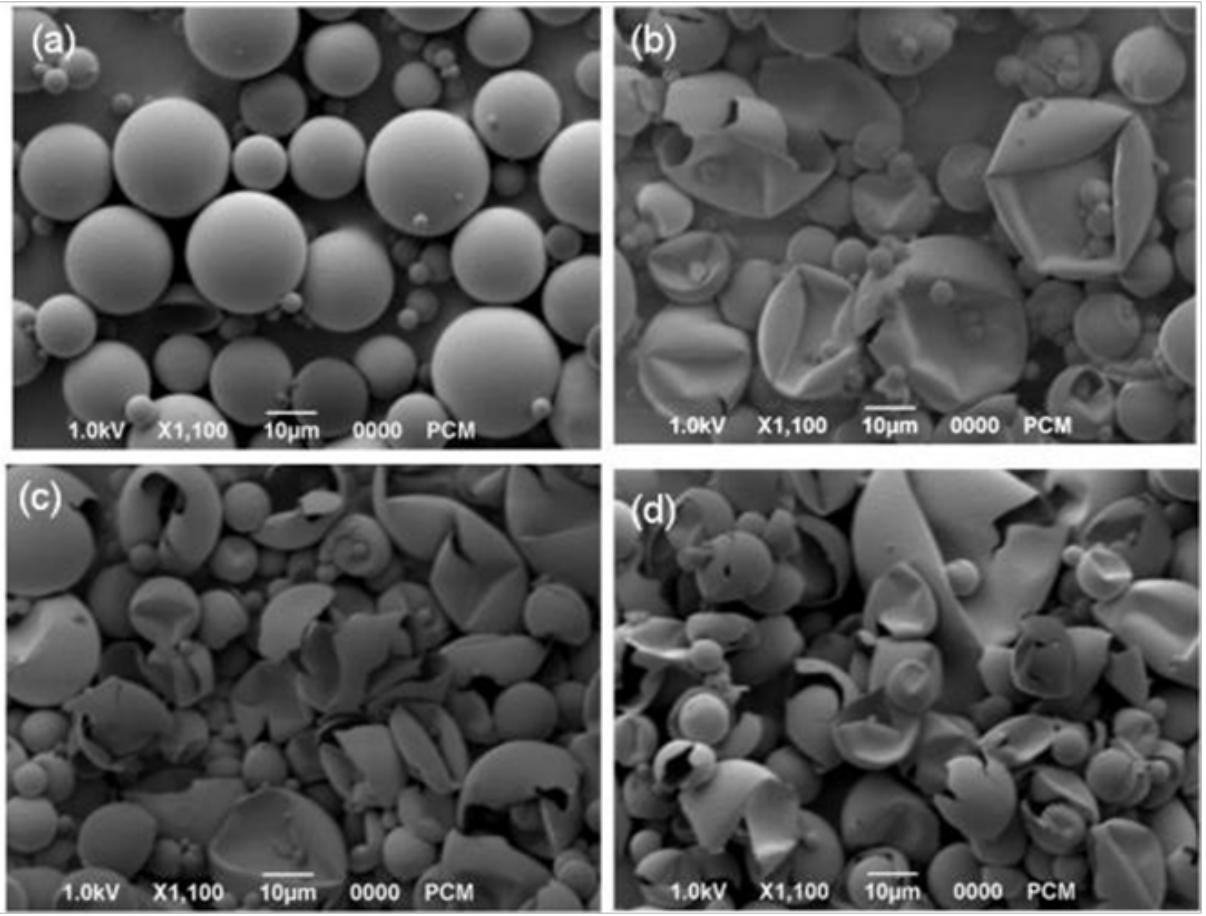


\subsubsection{Microtek 18D and Microtek 24D}

The SEM micrographs indicated that the two micro-encapsulated PCMs (Microtek 18D and 24D) underwent both collapse and rupture by 1680 cycles (Figures 4-6 and 4-7). It is believed that the many fragments of microcapsule shells in the post-cycled samples act as micro-impurities in the mixture of PCM that interfere with phase transformations. This interpretation is supported by the DSC data, as is the decrease in phase change temperature for Microtek 18D (with the low melting temperature), which is especially apparent when the pre- and post-cycled samples are compared.

Neither of the micro-encapsulated PCM materials were properly contained; they escaped from the microcapsules as the microcapsules ruptured, ultimately altering the physical and thermal properties of the Microtek PCMs. This behavior defeats the original purpose of the microcapsules, which was to contain PCM.

Figure 4-6. SEM images of BioPCM M51: (a) before thermal cycling, (b) after 1680 cycling (6.2 years), (c) after 3696 cycling (13.7 years), and (d) after 5400 cycling (20 years).
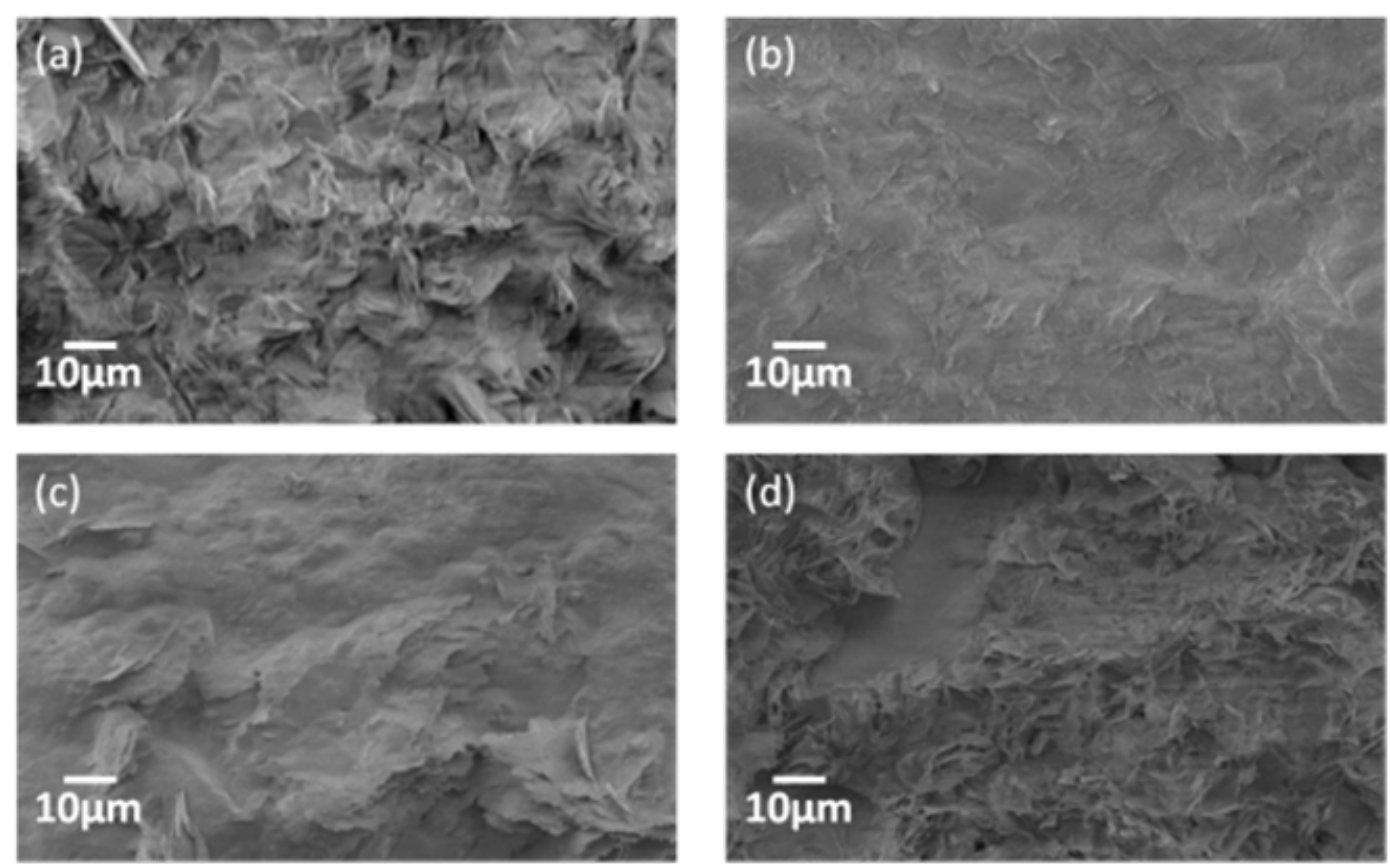
Figure 4-7. SEM images of Energain: (a) before thermal cycling, and (b) after 5400 cycling (20 years).
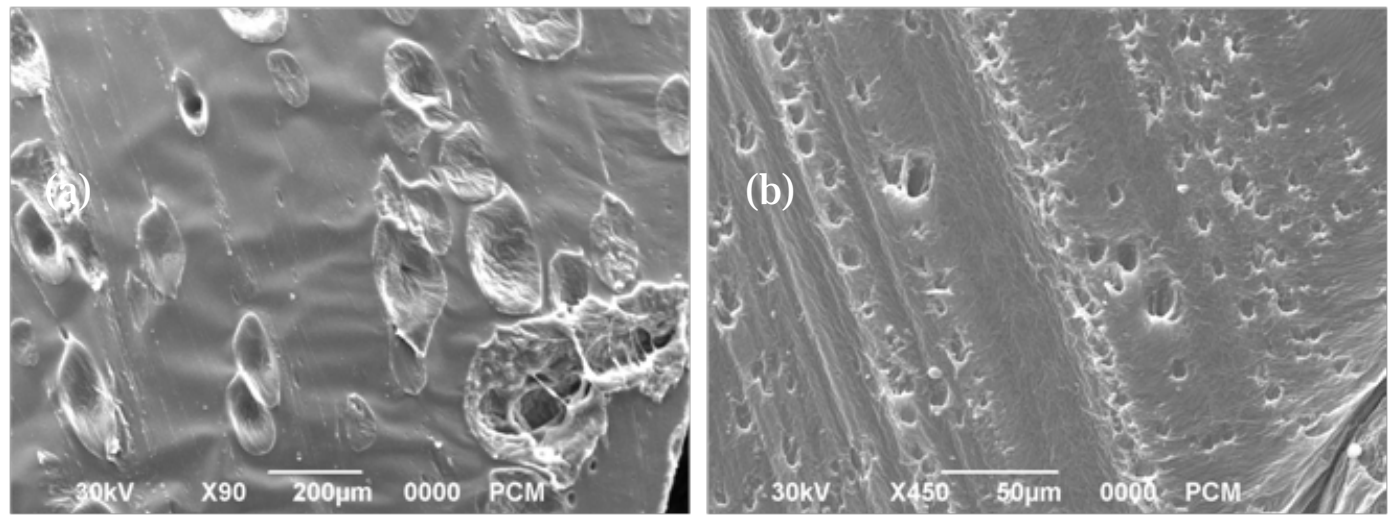

\subsection{Fourier Transform Infrared Spectroscopy (FTIR) results}

\subsubsection{BioPCM}

The long-term chemical stability of BioPCM M51 was analyzed using FTIR techniques. Figure 4-8a and b show the transmittance spectra of the BioPCM M51 after thermal cycling, overlayed with baseline scans. Table 4-5 lists the different peaks in the FTIR spectrum. It is known that BioPCM M51 is derived from underused feedstock such as soya oil, coconut oil, palm oil. These sources of oils are mostly composed of fatty acid such as $\alpha$-linolenic acid, oleic acid, and palmitic acid (Rohmana 2010). These fatty acids contain long hydrocarbon chains with $-\mathrm{CH}_{2}$ and $-\mathrm{CH}_{3}$ groups.

In the FTIR spectrum, the peaks at 2,926 cm-1, $2851 \mathrm{~cm}^{-1}, 719 \mathrm{~cm}^{-1}$, which are due to the stretching vibration of these groups, indicate the presence of long liner aliphatic chain in compound (Yu S. 2014). The peak at $1743 \mathrm{~cm}^{-1}$ represents the carbonyl group in oleic acid. The peaks in a range of 1600$1500 \mathrm{~cm}^{-1}$ and the peak at $1458 \mathrm{~cm}^{-1}$ indicate the presence of aromatic additives, which may also include fire retardants. The peaks at $1698 \mathrm{~cm}^{-1}$ and in a range from 1250-1000 $\mathrm{cm}^{-1}$ represent the stretching and stretching vibrations of $\mathrm{C}=\mathrm{O}$ and - $\mathrm{C}-\mathrm{O}$ groups presence in the fatty acid elements. The peak at $2327 \mathrm{~cm}^{-1}$, which could not be related to the BioPCM M51 PCM molecule structure, could be an additive with nitrile functional group. No changes were detected in the distribution of the peaks in the FTIR spectra. This indicates that BioPCM M51 PCM has good chemical stability when exposed to the extended thermal cycling. However there is noticeable disappearance of the small peak at $2327 \mathrm{~cm}^{-1}$ in spectrum after 3696 cycles, and after 5376 cycles; this probably indicates that there is a minor change in the PCM additive due to thermal cycling. 
Figure 4-8. FTIR spectra of BioPCM M51: (a) comparison among four cycles, and (b) comparison between 0 cycle and 5400 cycles.
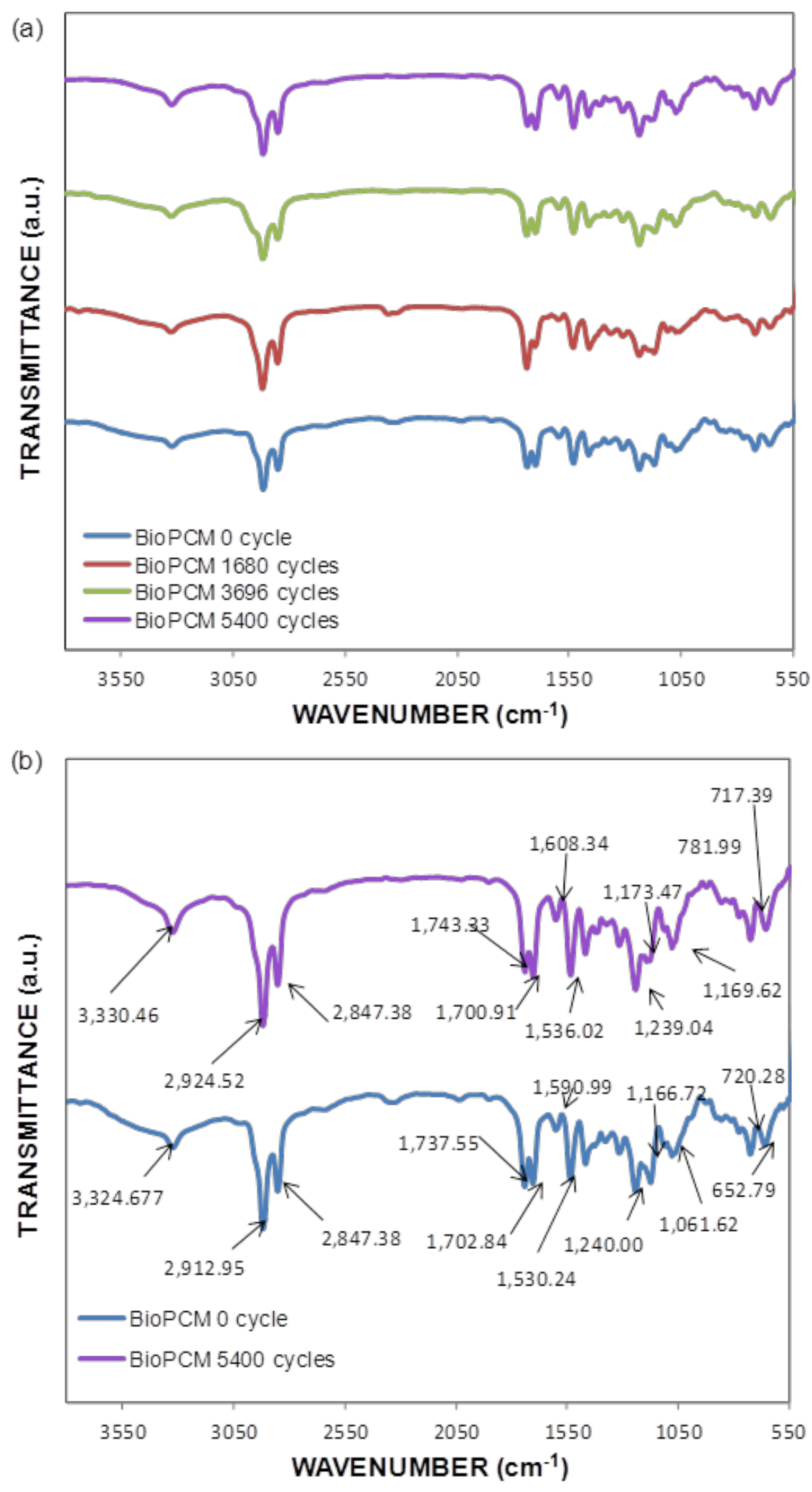
Table 4-5. FTIR spectrum peaks for BioPCM M51.

\begin{tabular}{|c|l|}
\hline Group frequency $\left(\mathbf{c m}^{-1}\right)$ & Assignment \\
\hline 3325.64 & - OH hydrogen bonding \\
\hline 2926.45 & Asymmetrical stretching vibration of $-\mathrm{CH}_{3}$ \\
\hline 2851.24 & Stretching vibration of $-\mathrm{CH}_{2}$ \\
\hline 2327.66 & C N stretch \\
\hline 1743.33 & C=C carbonyl \\
\hline 1698.02 & C=O Stretch \\
\hline $1600-1500$ & Presence of aromatic ring \\
\hline 1458.89 & C-C=C asymmetric stretch \\
\hline $1250-1000$ & $-\mathrm{C}-0$ stretch and stretching vibration \\
\hline 719.318 & $-\mathrm{CH}$ rocking vibration \\
\hline
\end{tabular}

\subsubsection{Energain}

According to the Material Safety Data Sheet (MSDS) provided by the manufacturer, the main ingredients in Energain are a paraffin and a co-polymer. Figure 4-9a and b show the FTIR spectra. Table 4-6 lists the different peaks in the FTIR spectrum. Baseline spectra of Energain show strong transmission peaks at 2921 and $2854 \mathrm{~cm}^{-1}$, which are known peaks of C-H stretching, indicating the presence of long liner chain of aliphatic hydrocarbon. From the intensity of this group of peaks, it can be concluded that most of the structure of this material is based on saturated and unsaturated hydrocarbons. Additionally, a sharp peak at the $1460 \mathrm{~cm}^{-1}$ is assigned to $\mathrm{C}-\mathrm{H}$ bending in the cyclic alkanes. The smaller peak at 1372 and $723 \mathrm{~cm}^{-1}$ is observed due to the $\mathrm{C}-\mathrm{H}$ in plane and out of plane bending mode. In addition to the hydrocarbon groups, there is a sharp peak at $1712 \mathrm{~cm}^{-1}$ due to the $\mathrm{C}=\mathrm{O}$ stretching of carbonyl groups and a band at $1090.55 \mathrm{~cm}^{-1}$ corresponding to the stretch of C-O bonds that are present in the material. No significant degradation in chemical stability was observed by this FTIR analysis. 
Figure 4-9. FTIR spectra of Energain: (a) comparison among four cycles, and (b) comparison between 0 cycle and 5400 cycles.
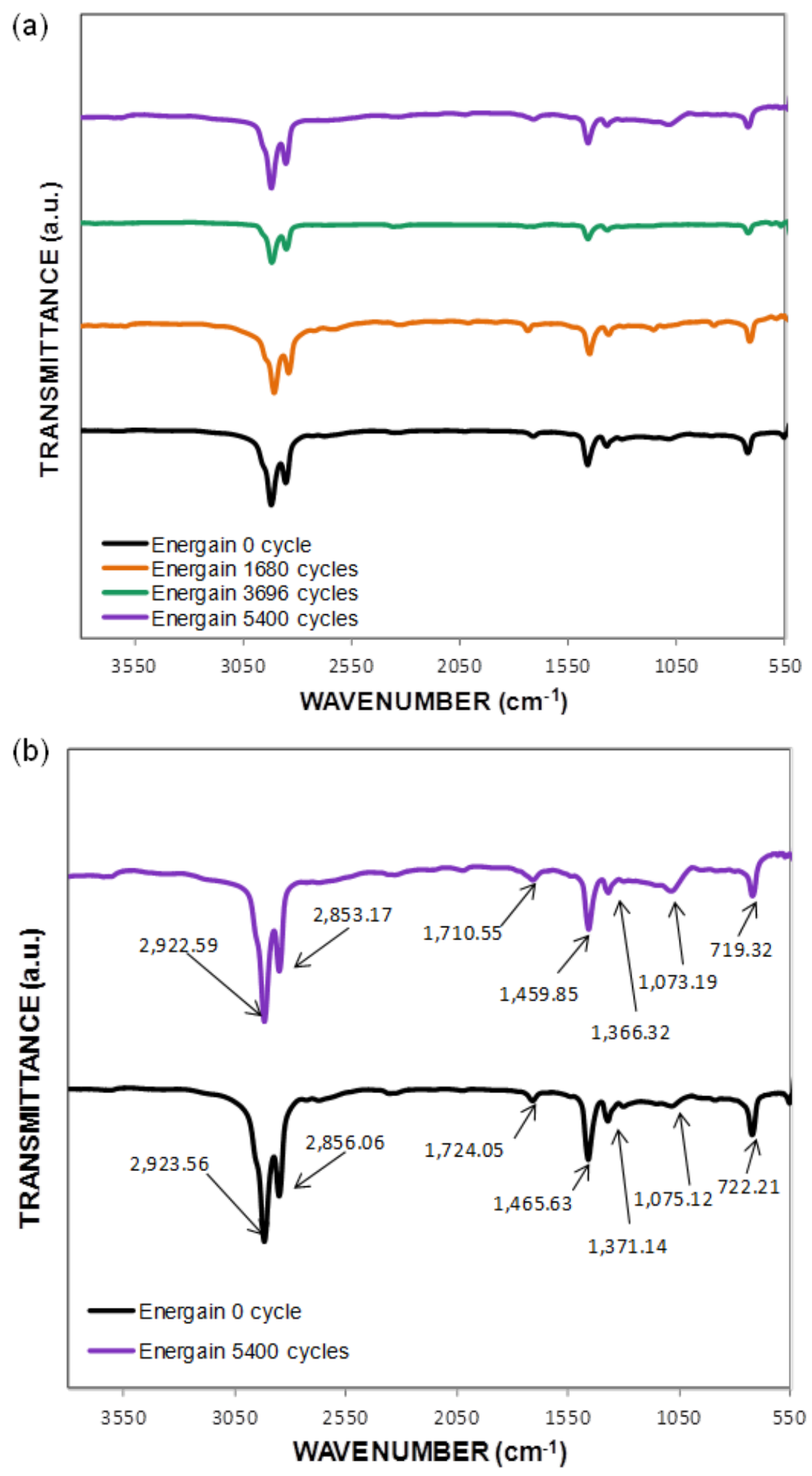
Table 4-6. FTIR spectrum peaks for Energain.

\begin{tabular}{|c|l|l|}
\hline Wave number & Assigned group & Changes after thermal treatment \\
\hline 3615.88 & O-H free hydroxyl group & $\begin{array}{l}\text { Prominent with increased number of thermal } \\
\text { cycles }\end{array}$ \\
\hline 2921.63 & C-H stretch Alkanes & No change \\
\hline 2854.13 & C-H stretch Alkanes & No change \\
\hline 2679.6 & O-H in carboxylic acid & Not found in baseline \\
\hline 1712.48 & C=O stretch Maleic anhydride & Broaden with increased number of cycles \\
\hline 1460.81 & C-H bending in alkanes & Shorten with increased number of cycles \\
\hline 1372.1 & C-H in plane out plane bending & Shorten \\
\hline 1090.55 & C-O stretch & No change \\
\hline 952.66 & O-H bend in carboxylic acid & Not found in baseline \\
\hline 723.17 & C-H rock in Alkanes & No change \\
\hline
\end{tabular}

\subsubsection{Microtek 18D and Microtek 24D}

Figures 4-10 and 4-11 show the FTIR spectra for Microtek 18D and 24D samples. Table 4-7 lists the different peaks in the FTIR spectrum. According to manufacturer's MSDS, the PCM core material of Microtek 18D and Microtek 24D is octadecane paraffin wax. In both PCMs, the peaks at 2921 $\mathrm{cm}^{-1}, 2857 \mathrm{~cm}^{-1}, 1437 \mathrm{~cm}^{-1}$ and $719 \mathrm{~cm}^{-1}$ in baseline are assigned to $\mathrm{CH}_{2}$ and $\mathrm{CH}_{3}$ stretching and bending of aliphatic hydrocarbon. The peak in the range of 1600 to $1500 \mathrm{~cm}^{-1}$ represents the C-C stretching vibration of aromatic ring. This indicates that PCM was encapsulated in a polymer, which might have aromatic rings in structure.

After thermal cycling, the FTIR spectra of 18D showed new peaks at 3345 $\mathrm{cm}^{-1}$ and $1133 \mathrm{~cm}^{-1}$, which appear to be attributed to N-H stretching of amine group and $\mathrm{C}-\mathrm{N}$ aliphatic amine stretching. This indicates that some chemical reactions might occur in the early stages of thermal cycling. In the Microtek 24D FTIR spectra, the peak at $1009 \mathrm{~cm}^{-1}$, which is assigned to - $\mathrm{C}-\mathrm{H}$ bending of alkenes, becomes prominent after thermal cycling. This indicates that Microtek 24D is more stable than the Microtek 18D. 
Figure 4-10. FTIR spectra of Microtek 18D: (a) among four cycles, and (b) between 0 cycle and 5400 cycles.
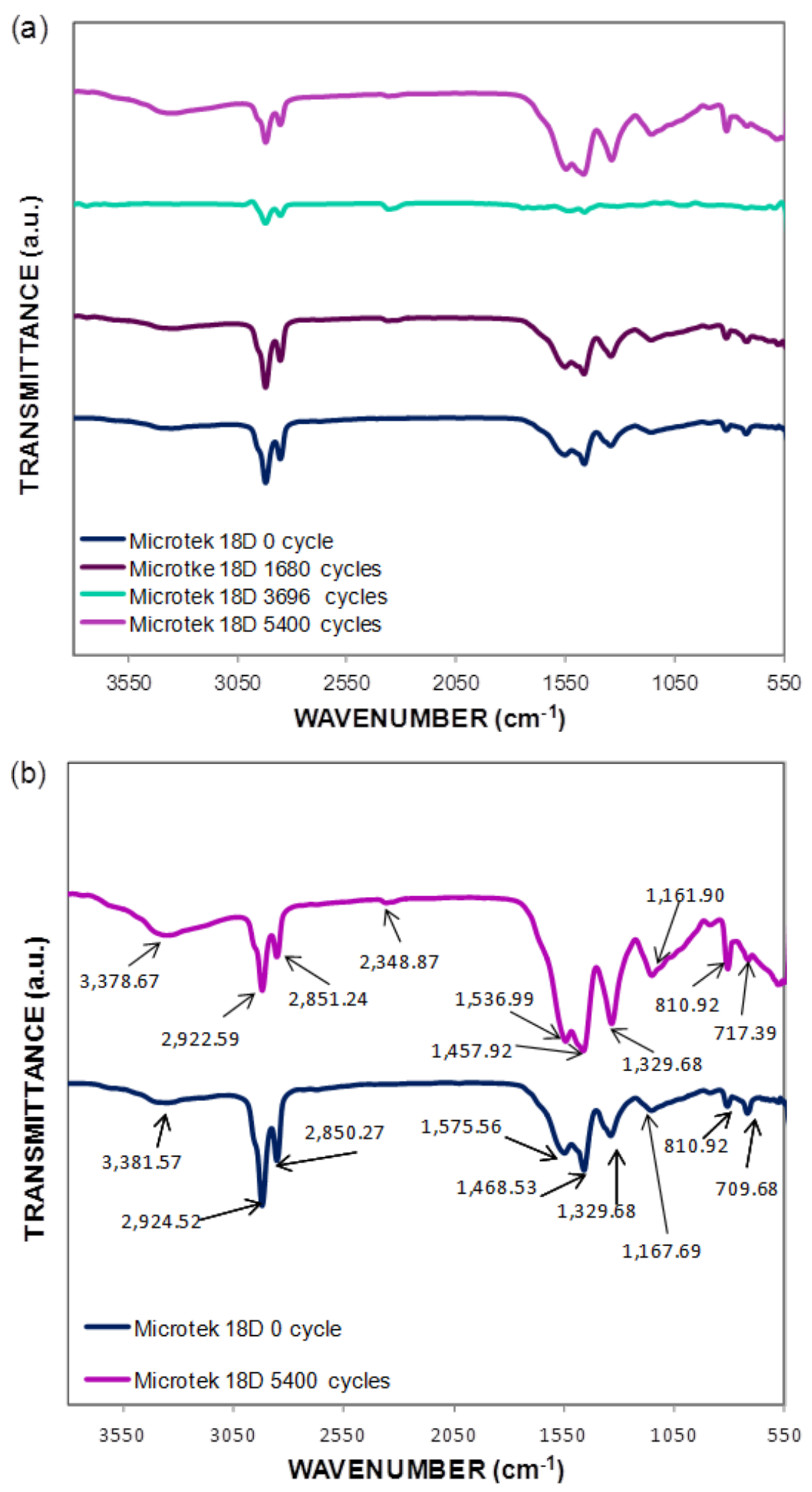
Figure 4-11. FTIR spectra of Microtek 24D: (a) among four cycles, and (b) between 0 cycle and 5400 cycles.
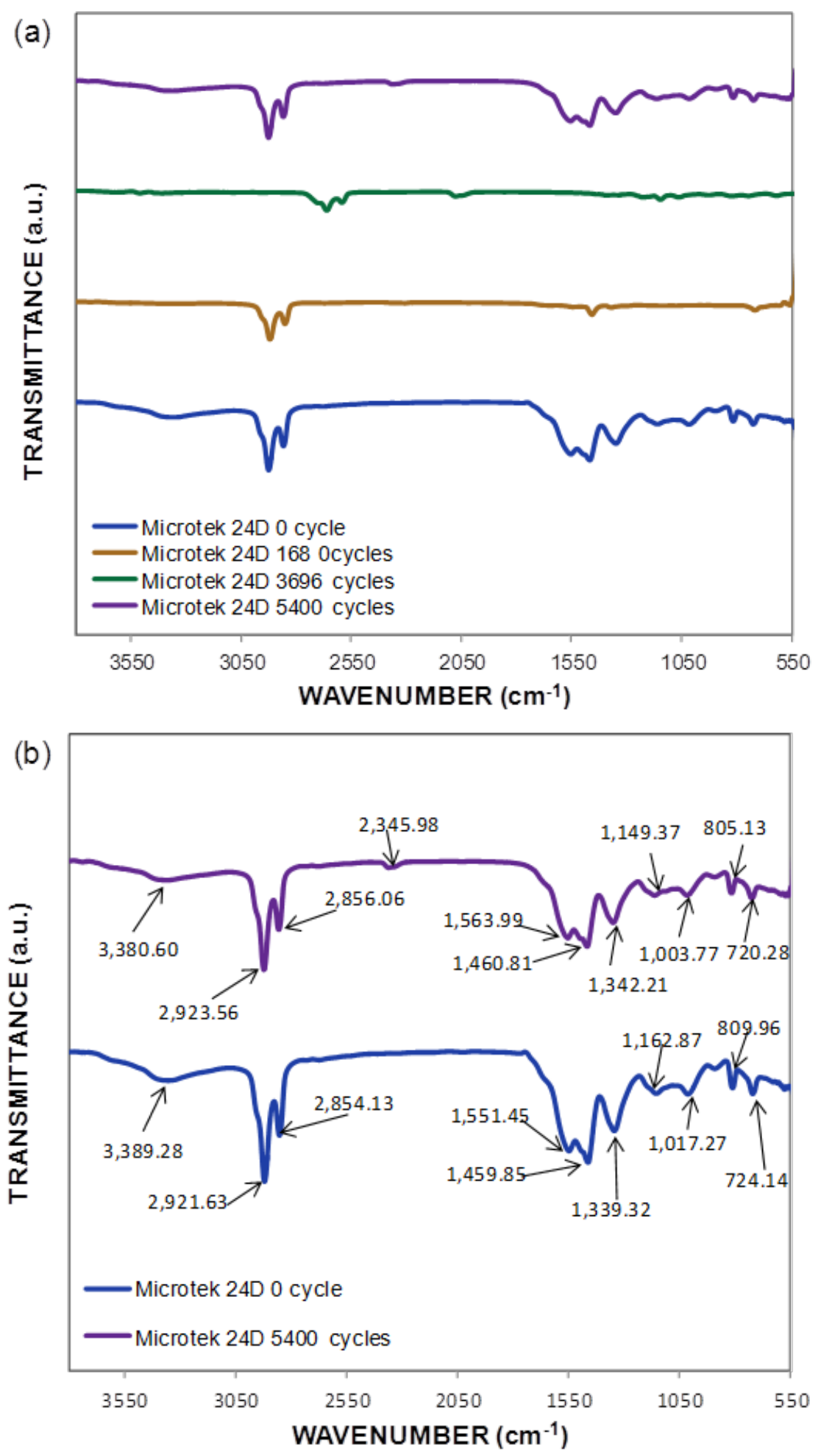
Table 4-7. FTIR spectrum peaks for Microtek 18D and 24D.

\begin{tabular}{|c|l|c|l|}
\hline Group frequency $\left(\mathrm{cm}^{-1}\right)$ & Assignment & Group frequency $\left(\mathrm{cm}^{-1}\right)$ & Assignment \\
\hline 719.318 & $-\mathrm{CH}_{2}$ rocking vibration & 1558.2 & C-C ring stretching vibration \\
\hline 1009 & C-H bend of alkenes & 2857.02 & $-\mathrm{CH}_{3}$ vibration stretch \\
\hline 1133.94 & C-N aliphatic amine stretch & 2921.63 & $\mathrm{CH}_{2}$ vibration stretch \\
\hline 1473.35 & $\mathrm{CH}$ bending & 3345.89 & $\mathrm{~N}-\mathrm{H}$ stretch of amine group \\
\hline
\end{tabular}

\subsection{TGA results}

\subsubsection{BioPCM}

Thermogravimetric analysis (TGA) was used to investigate the decomposition of BioPCM M51 after 1680, 3696 and 5376 thermal cycles and to compare the results with baseline (unexposed) samples. Figure 4-12a and b show the TGA results. In Figure 4-12a, the solid line curves represent TGA and the dotted line curves represent first order Derivative of TGA (DrTGA). For the purposes of further discussion, the TGA curves have been divided into three zones, which define the range of temperatures where the weight loss occurred in TGA experiment. The temperature zones 1,2 , and 3 ranged from $212-491^{\circ} \mathrm{F}\left(100-255^{\circ} \mathrm{C}\right), 491-563^{\circ} \mathrm{F}\left(255-295^{\circ} \mathrm{C}\right)$, and $563-752^{\circ} \mathrm{F}\left(295-400{ }^{\circ} \mathrm{C}\right)$, respectively.

In the baseline samples the highest weight loss occurred at $474^{\circ} \mathrm{F}$ $\left(245.6^{\circ} \mathrm{C}\right)$. The unexposed BioPCM M51 thermal decomposition onset temperature occurred:

- over the range of 404.3 to $405.4^{\circ} \mathrm{F}$ (206.82 to $207.42^{\circ} \mathrm{C}$ ) for 1680 thermal cycles,

- at $397.4^{\circ} \mathrm{F}\left(203^{\circ} \mathrm{C}\right)$ for 5376 thermal cycles, and

- at $394.8^{\circ} \mathrm{F}\left(201.53^{\circ} \mathrm{C}\right)$ for 5376 thermal cycles.

The onset temperature in Zone 2 and 3 is $510.67^{\circ} \mathrm{F}\left(265.9^{\circ} \mathrm{C}\right)$ and $567.2^{\circ} \mathrm{F}\left(297.3^{\circ} \mathrm{C}\right)$, respectively, for the baseline sample. The decomposition temperature in Zone 2 is noticeably lower than that in Zone 3, from 510.7 to $477.6^{\circ} \mathrm{F}$ ( 265.9 to $247.6^{\circ} \mathrm{C}$ ) at 5400 cycles. In Zone 3, the onset temperature is more stable, in range of 567.014 to $559.85^{\circ} \mathrm{F}$ ( 297.2 to $293.3^{\circ} \mathrm{C}$ ) for the given cycle times. This indicates that some decomposition that has taken place in Zone 2, which could be attributed to the additives used to enhance the thermal properties. Overall, the TGA results suggested that the BioPCM M51 has good thermal stability in the laboratory simulated environments. In addition, BioPCM did not show significantly change after having been exposed to over 5,400 thermal cycles. 
Figure 4-12. TGA results for BioPCM.

(a) TGA

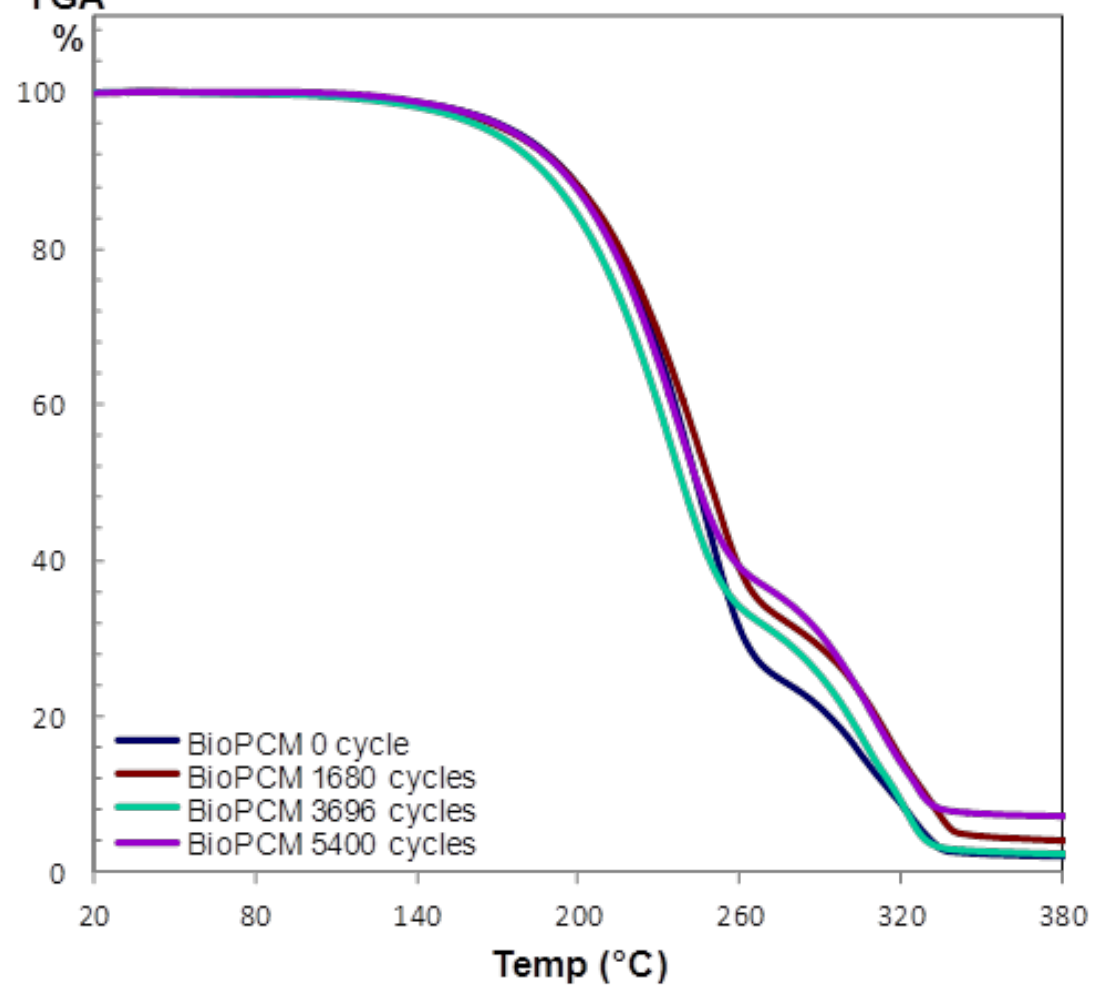

(b) TGA

DrTGA

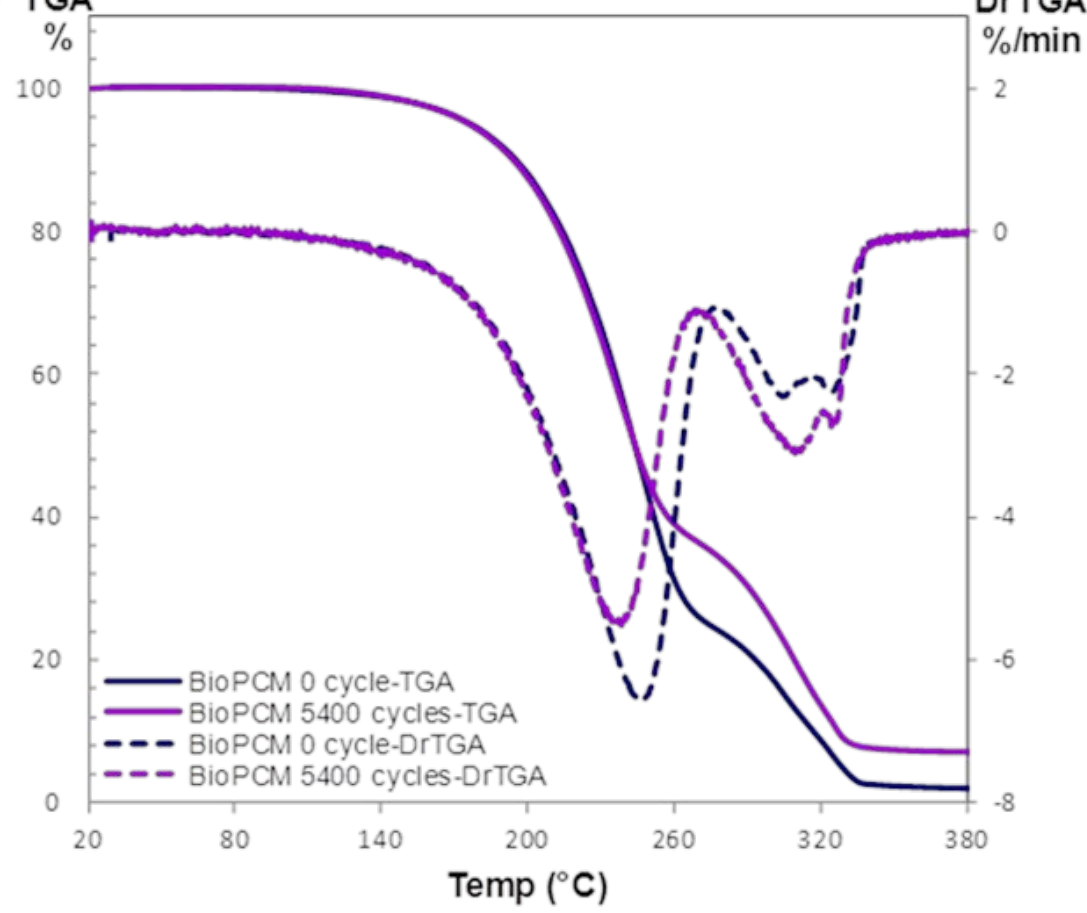




\subsubsection{Energain}

Figure 4-13a and $b$ show the TGA analysis for Energain PCM. The results indicate a noticeable difference in material thermal behavior after the material has undergone 3696 phase change cycles. Also, there appears to be a greater moisture entrapment in the baseline sample that evaporated and resulted in greater weight loss in the temperature range of $212-392^{\circ} \mathrm{F}$ (100-200 $\left.{ }^{\circ} \mathrm{C}\right)$. The residual \% weight in the baseline sample is lower than the residual weight loss for samples exposed to 3696 cycles, indicating a possible greater amount of thermally decomposable material in baseline sample. The rate of weight loss also varies, as can be seen see after 3696 cycles, but the rate of weight loss is relatively slower at initial stages of decomposition. This analysis did not indicate significant degradation of Energain as a result of thermal cycling.

A comparison of DSC and TGA results for Energain subjected to 1680 cycles show that, while both depart from the pre-cycled Energain sample behavior, the DSC shows a loss of latent heat and a significant shift in freezing point temperature. After 3696 cycles, the TGA also shows that the material decomposition profile has changed from the pre-cycled profile, which is similar to that seen at 1690 cycles; however, according to DSC, the PCM has begun to recover its pre-cycled latent heat value. Eventually, the PCM recovers its pre-cycled latent heat value (at 5400 cycles), and the transition temperatures are close to the pre-cycled values, as is also seen in the TGA decomposition profile. These anomalous results may be attributed to influence of the co-polymer component playing a more significant role in the behavior of the paraffin-co-polymer compound during these phases of cycling. 
Figure 4-13. TGA analysis for Energain PCM.

(a) TGA

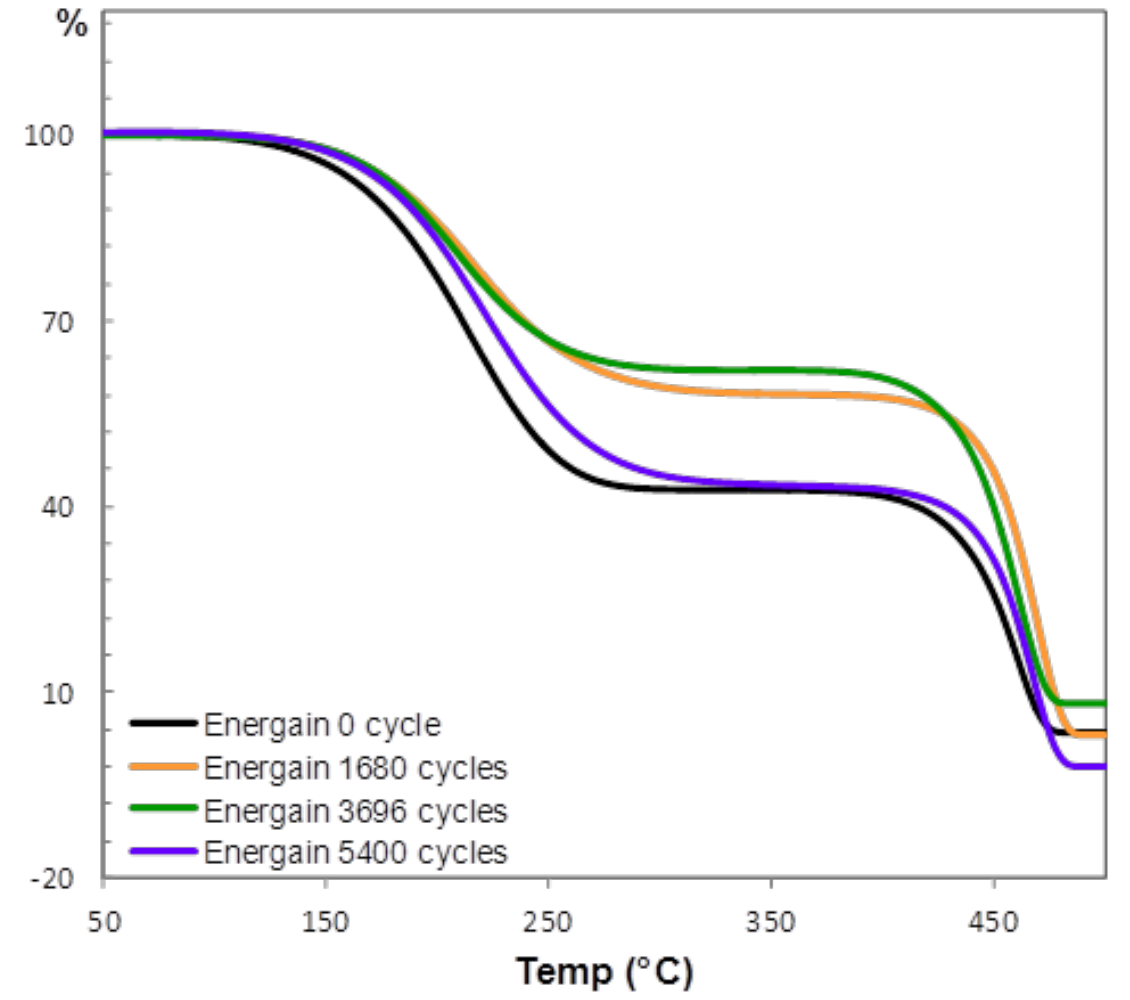

(b) TGA DrTGA

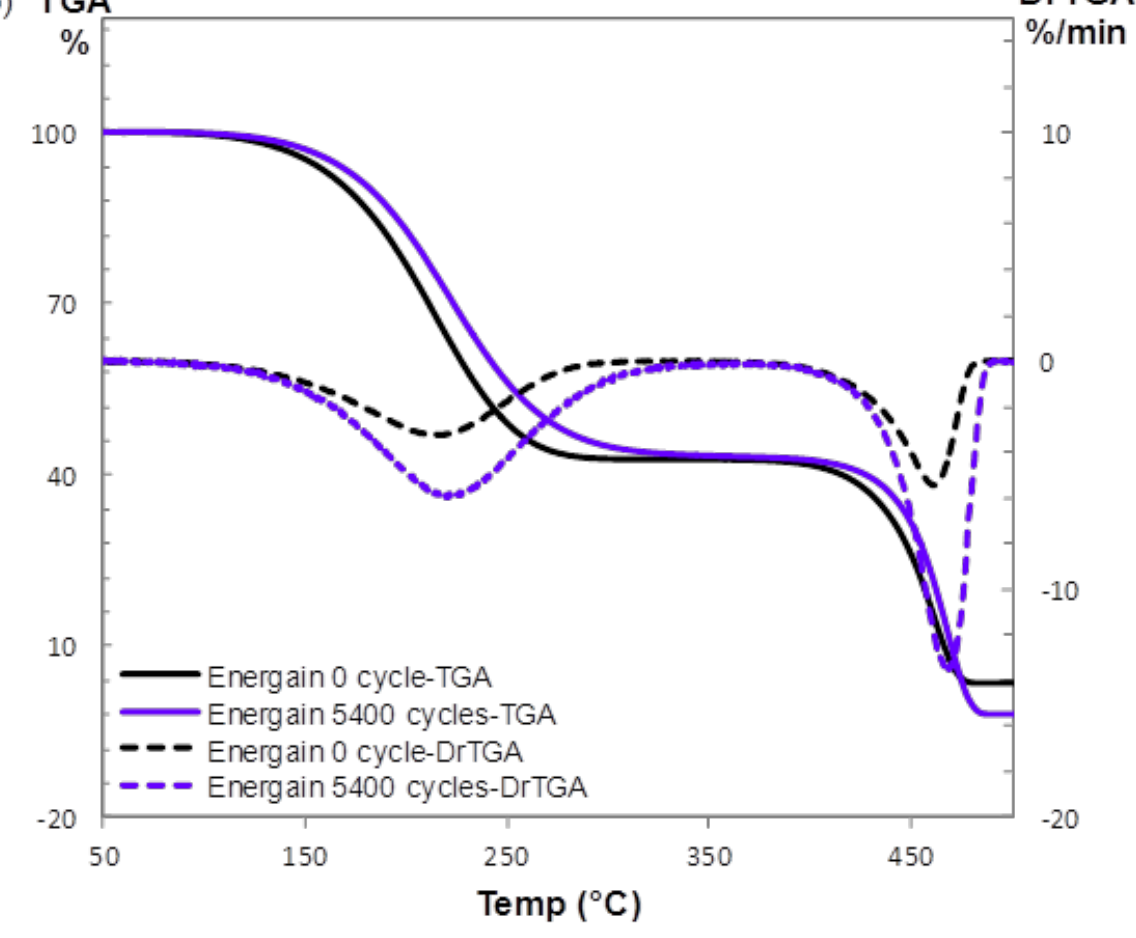




\subsubsection{Microtek 18D and Microtek 24D}

Figures 4-14a and $\mathrm{b}$ and 4-15a and $\mathrm{b}$ show the TGA analyses for Microtek 18D and Microtek 24D, respectively. In the unexposed (baseline) samples, the greatest weight loss occurred at $729.4^{\circ} \mathrm{F}\left(387.4^{\circ} \mathrm{C}\right)$ for Microtek 18D and at $704.8^{\circ} \mathrm{F}\left(373.8^{\circ} \mathrm{C}\right)$ for Microtek 24D.

The baseline TGA curve for Microtek 24D shows gradual weight loss in a range from $527-797^{\circ} \mathrm{F}\left(275-425^{\circ} \mathrm{C}\right)$, which indicates that the microcapsule shell was composed of more than one monomer. The baseline TGA curve of the Microtek 18D shows an abrupt drop in weight loss at the decomposition temperature, which indicates that its microcapsule was made from a single monomer. However both PCMs showed the same decomposition trend after 1680 cycles. The thermal decomposition temperatures unexpectedly dropped and the greatest percent weight loss in both PCMs occurred in the temperature range from 309.2 to $320^{\circ} \mathrm{F}$ ( 154 to $160{ }^{\circ} \mathrm{C}$ ). However, after first drastic decomposition in the TGA, the PCMs became stable in later cycles. This indicates that the encapsulated PCM probably escaped in greater amounts in the 18D micro-encapsulated samples due to degradation of coating polymer after a certain number of cycles.

For both the Microtek 18D and 24D samples, TGA indicated that the decomposition temperature has shifted from 752 to $428^{\circ} \mathrm{F}$ ( 400 to $220^{\circ} \mathrm{C}$ ), which was attributed to the rupturing of the microcapsules containing the PCM, as shown by SEM analysis. The microcapsule polymeric shells decompose at $716^{\circ} \mathrm{F}\left(380^{\circ} \mathrm{C}\right)$, as shown in the TGA curves for the pre-cycled sample (0 cycles), whereas the post-cycled TGA decomposition temperature are close to that of the decomposition temperatures of the PCM materials (hexadecane / octadecane) within the microcapsule. In baseline samples, the shells that microencapsulate the PCM are intact, and the decomposition temperature occurs at $752^{\circ} \mathrm{F}\left(\sim 400^{\circ} \mathrm{C}\right)$. After 1800 cycles, much of the PCM sample had apparently leaked out of the shells, and since it is no longer protected by the shells, the decomposition temperature occurs at a much lower temperature. 
Figure 4-14. TGA analyses for Microtek 18D.

(a) TGA

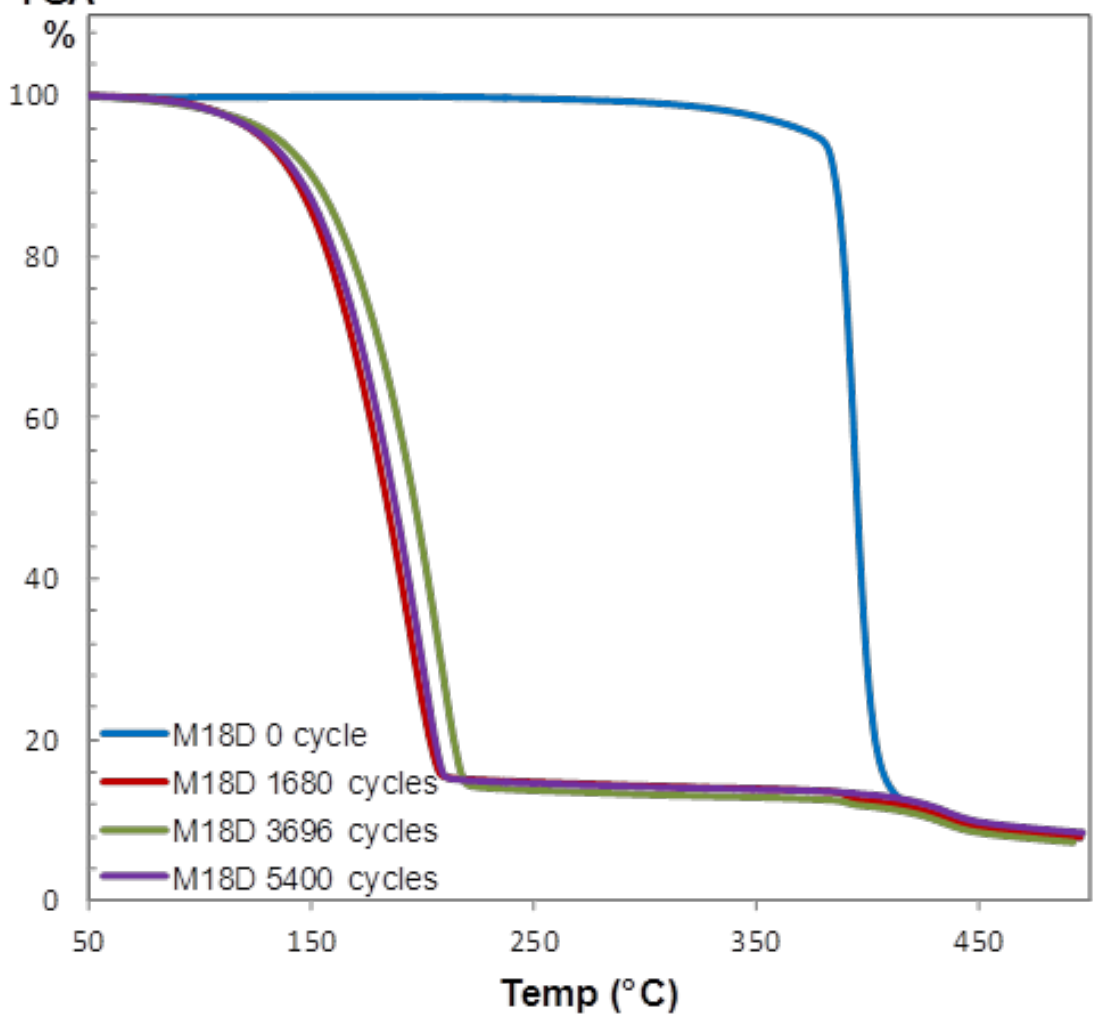

(b) TGA

DrTGA

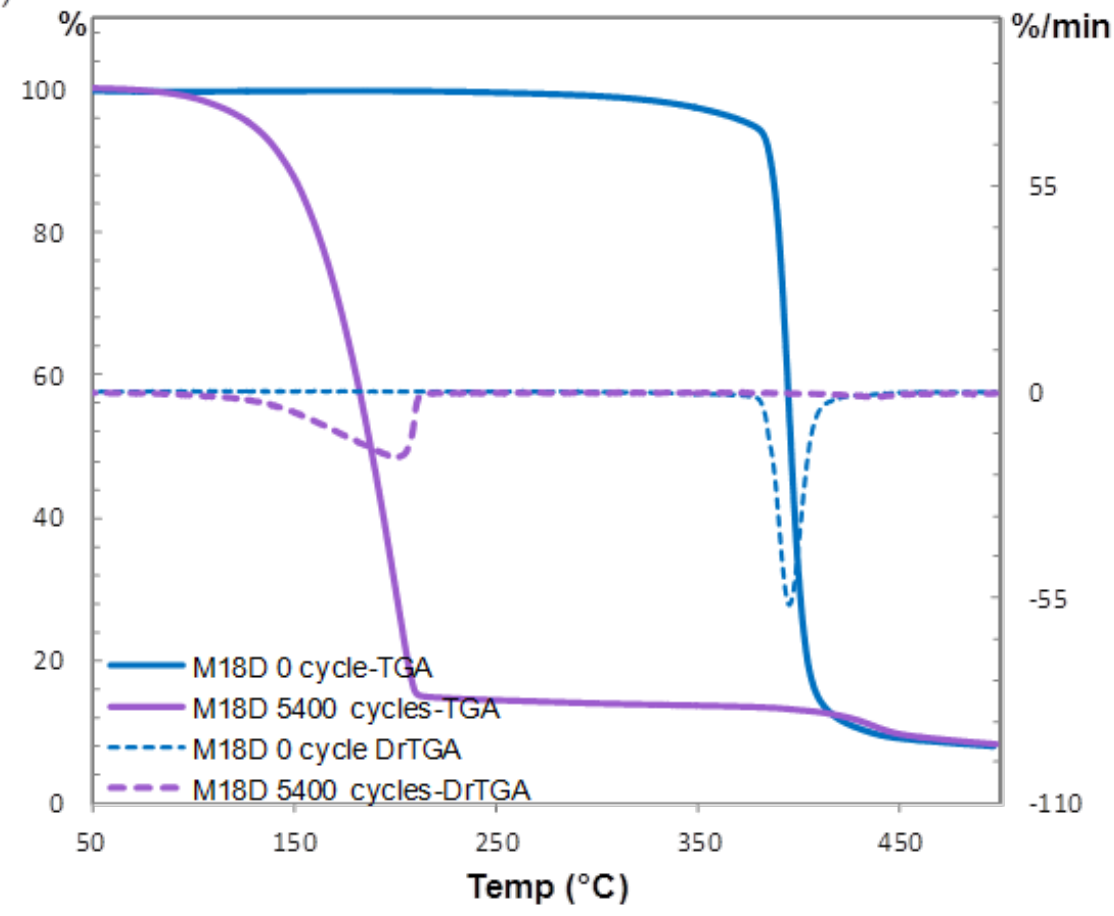


Figure 4-15. TGA analyses for Microtek 18D and Microtek 24D...

(a) TGA

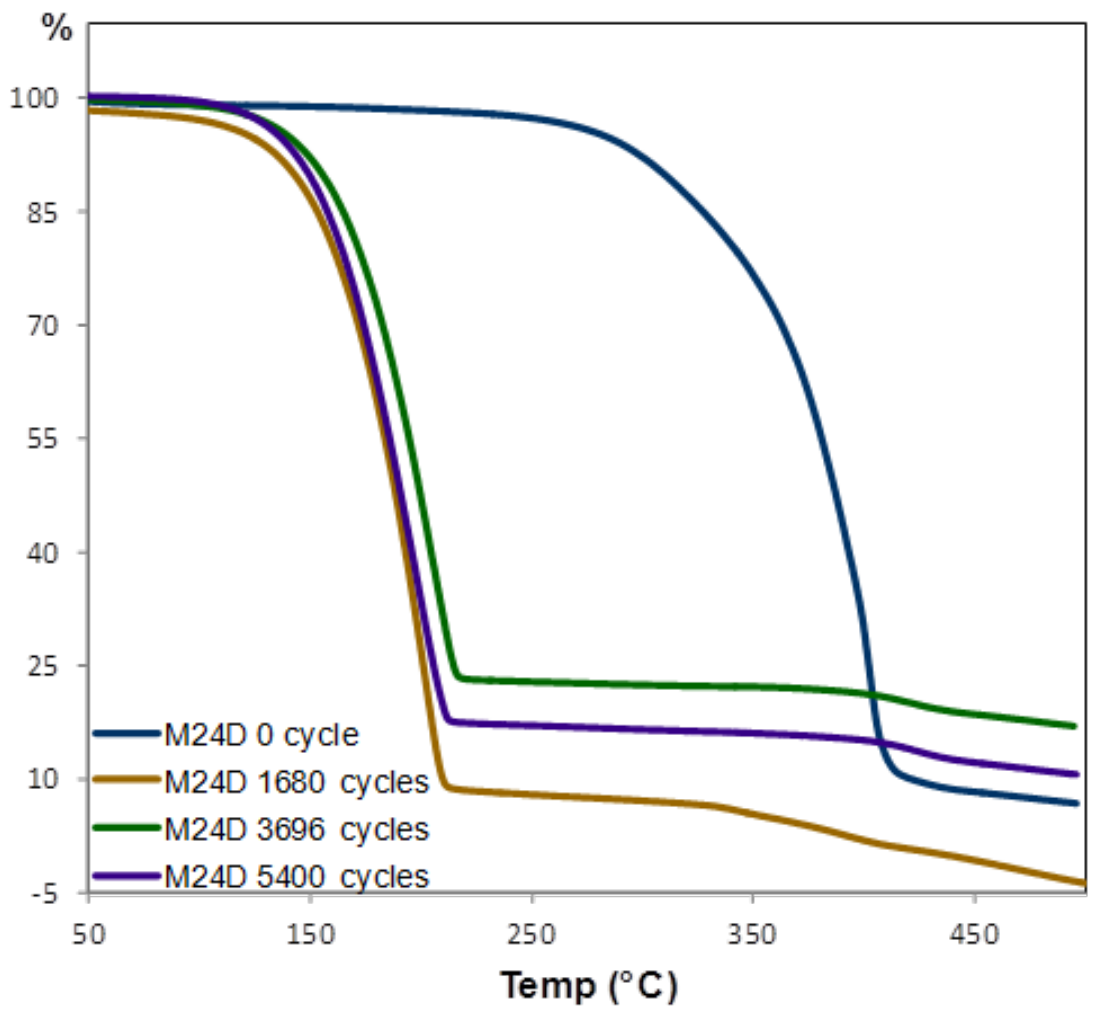

(b) TGA

DrTGA

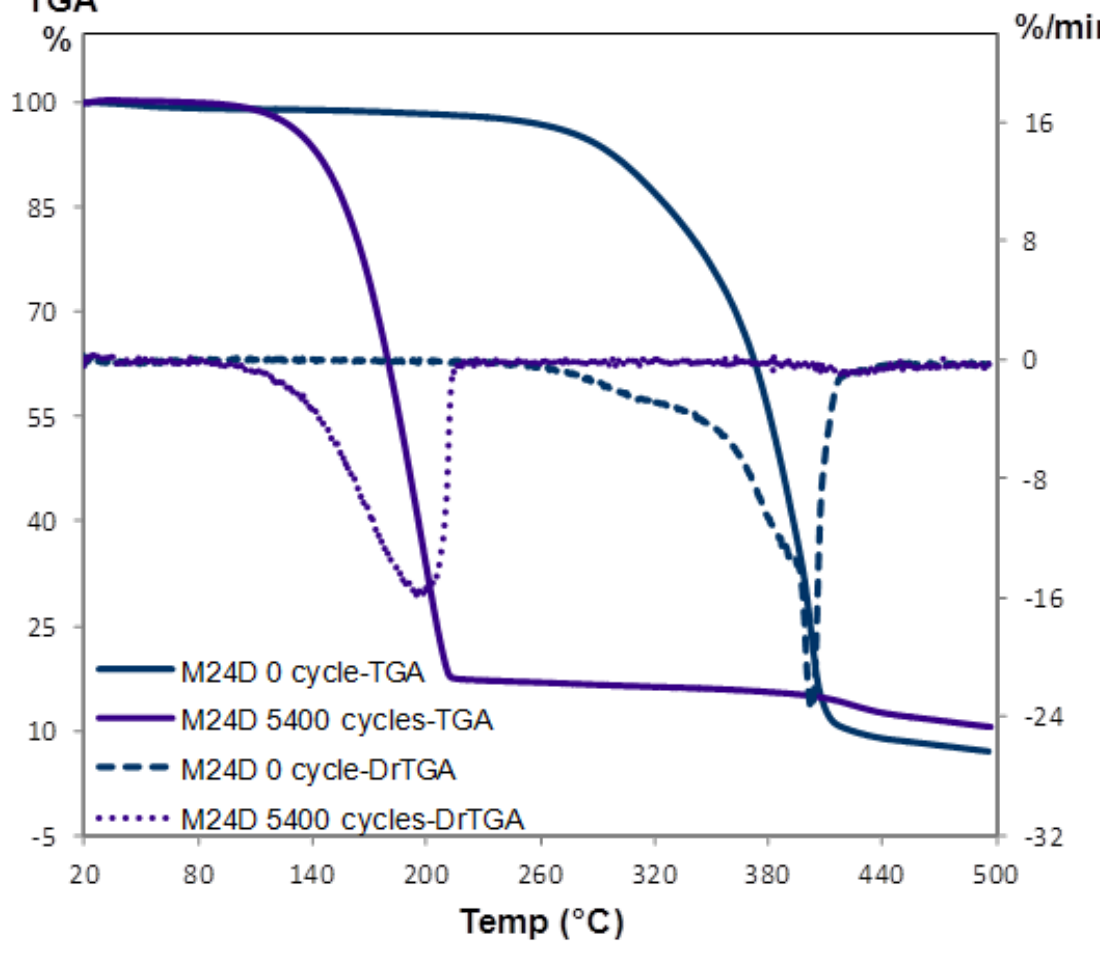




\section{Conclusion and Recommendations}

This work conducted thermal cycling experiments to establish a standard protocol for evaluation of the long-term performance of PCMs using relatively short term tests. Four commercially available PCM s were subjected to up to 5,400 cycles of thermal cycling at 90 minutes per cycle, over the temperature range of $14-140{ }^{\circ} \mathrm{F}\left(-10-60^{\circ} \mathrm{C}\right)$, to simulate 20 years of use in building envelopes: (1) BioPCMat ${ }^{\mathrm{TM}}$ M51, (2) DuPont ${ }^{\mathrm{TM}}$ Energain $^{\circledR}$ Ther- $^{-}$ mal mass panel, and $(3,4)$ MPCM with two different melting and freezing points. At 3-4 week time intervals, the samples were taken from the thermal cycling chamber and SEM micrographs, DSC, TGA, and FTIR data were obtained and compared to baseline (pre-cycling data). The results have indicated that baseline latent heat values and freezing and melting transition temperatures were generally in accordance with the manufacturer's stated values. All PCM materials evaluated tended to lose some latent heat storage capacity as they underwent thermal cycling.

For BioPCM M51, the DSC results indicated that the measured latent heat for baseline samples was in accordance with the manufacture's MSDS. However, the latent heat decreased by $12-15 \%$ after 1680 cycles, which indicates that some degree of thermal degradation can be expected after 6 years of service in an operational environment. Thermal stability of the material (based on TGA analysis) did not show significant change in onset temperature. Also, there was no change detected in the peaks distribution in the FTIR spectra, indicating that BioPCM M51 has good chemical stability against the extended thermal cycling. This indicates that most of the loss in the latent heat could be due to poor nucleation during the freezing cycle.

For Energain, the DSC results showed anomalous behavior at 1680 cycles, with significant increases in transition onset temperatures on the freezing cycle, as well as decrease in latent heat at 1680 cycles. These results were consistent with TGA, which showed a marked difference in the decomposition profile of the PCM compound at 1680 and 3696 cycles. Further cycling shows recovery of pre-cycled latent heat (with a slight increase), while TGA showed that the material eventually recovers its pre-cycled TGA decomposition profile. These anomalous results may be attributed to influence of the co-polymer component playing a more significant role in the behavior of the paraffin-co-polymer compound during these stages of thermal cycling. SEM micrographs of Energain PCM samples showed evidence of melting and re- 
crystallization; however, there was no significant damage or impurities observed in these micrographs. No significant degradation in chemical stability for Energain was indicated by this FTIR analysis.

Tests of Microtek 18D and 24D indicated that both types of microcapsules, containing the PCM compounds were severely damaged after only 1,680 thermal cycles, which strongly supports the conclusion that the PCM escaped from the ruptured microcapsules. DSC analysis showed a 15-23\% loss in latent heat capacity, and depression of the melting point of the $64.4^{\circ} \mathrm{F}\left(18^{\circ} \mathrm{C}\right)$ samples by up to $7.2^{\circ} \mathrm{F}\left(4^{\circ} \mathrm{C}\right)$. The $75.2^{\circ} \mathrm{F}\left(24^{\circ} \mathrm{C}\right)$ microencapsulated PCM (Microtek 24D) samples showed a much smaller reduction in latent heat capacity, about $8 \%$ loss, with no change in melting point. However, in both cases, TGA indicated that the microcapsules were damaged, which is consistent with SEM observations.

These results indicate that it is likely that the micro-encapsulated PCMs tested are too fragile to withstand the effects of over 1,500 thermal cycles over the range of $14-140{ }^{\circ} \mathrm{F}\left(-10-60^{\circ} \mathrm{C}\right)$. This observation calls into question the wisdom of using micro-encapsulated PCM, although it is possible that in actual applications, the micro-encapsulated PCM would not experience such a wide temperature range as was employed in this testing protocol. Alternatively, manufacturers may be able to develop improved formulations of micro-encapsulated PCMs that have more robust microcapsules shells to better contain the PCM.

This work concludes that thermal cycling in conjunction with DSC, SEM, and TGA appears to be a valid way to predict the long-term physical and thermal changes in a PCM samples based on short term testing. The 90minute testing cycle appears to be adequate for the purpose of quickly simulating daily phase changes that the PCM would experience in operational environments. It is noted that the high and low temperatures (140 and $14^{\circ} \mathrm{F}$ [60 and $\left.-10^{\circ} \mathrm{C}\right]$, respectively) used in the thermal cycling protocol in these experiments may be too severe to properly simulate most real-world applications, thus it may be necessary to modify them in further developments of the protocol. High and low temperatures of 32 and $104^{\circ} \mathrm{F}(40$ and $0{ }^{\circ} \mathrm{C}$ ) may prove to be adequate for this purpose. Such a modification would also allow faster accelerated testing using the same temperature ramp rate. 


\section{References}

American Society for Testing and Materials (ASTM). 1995. Standard Test Methods for Water Vapor Transmission of Materials. ASTM E96-95 (Method B). West Conshohocken, PA: ASTM International.

2009. Standard Method for Surface Burning Characteristics of Building Materials. ASTM E84-09. West Conshohocken, PA: ASTM International.

2011. Standard Test Method for Determining Specific Heat Capacity by Differential Scanning Calorimetry. ASTM E1269-11. DOI: 10.1520/E1269-11. West Conshohocken, PA: ASTM International.

Castellon, C., M. Medrano, J . Roca, M. Nogues, A. Castell, and L. F. Cabeza. 2007. Use of Microencapsulated Phase Change Materials in Building Applications. Atlanta, GA: American Society of Heating, Refrigerating, and Air-Conditioning Engineers (ASHRAE), http://www.solaripedia.com/files/991.pdf

Chung, O., S. G. J eong, S. Yu,and S. Kim. 2014. Thermal performance of organic $\mathrm{PCMs}$ / micronized silica composite for latent heat thermal energy storage. Energy and Building 70:180-185.

E. I. duPont de Nemours and Company, http://energain.co.uk/Energain/en_GB/index.html

Gao, E., B. Zhang, L. D. Stephenson, V. Boddu, and J. Trovillion. 2013. Prediction of phase change material (PCM) degradation. Conference Proceedings of the Thermal Performance of Exterior Envelopes of Whole Buildings XII, December 2013. Atlanta, DA: ASHRAE.

Günther, E., S. Hiebler, and H. Mehling. 2006. Determination of the heat storage capacity of PCM and PCM-objects as a function of temperature. Proceedings of the Tenth International Conference of Thermal Energy Storage. Stockton, NJ : Stockton University, http://intraweb.stockton.edu/eyos/energy_studies/content/docs/FINAL_PAPERS/11B-2.pdf

Kosny, J., D. W. Yarbrough, I. Rizaai, D. Keuthold, J . B. Smith, and M. Bianchi. 2009. Development and testing of ignition resistant microencapsulated phase change material. Proceedings of the $11^{\text {th }}$ International Congress on Thermal Energy Storage (Effstock), Stockholm, Sweden 14-17J une, 2009, http://intraweb.stockton.edu/eyos/energy_studies/content/docs/effstock09/Session_4_2_PC $\underline{M}$ in_Buildings/33.pdf

PhaseChange Energy Solutions. 2014. Web site, http://www.phasechange.com/index.php/en/

Microtek Laboratories, Inc. 2015. Microencapsulated PCMs. Web site. Dayton, OH, http://www.microteklabs.com/micropcm.html

Rohmana, A., Y. B. Che Man. 2010. Fourier transform infrared (FTIR) spectroscopy for analysis of extra virgin olive oil adulterated with palm oil. Food Research International 43(3):886-892. 
Royon, L., G. Guiffant, and P. Flaud. 1997. Investigation of heat transfer in a polymeric phase change material for low level heat storage. Energy Conversion and Management 38(6):517-524

Sharma, A., V. V. Tyagi, C. R. Chen, and D. Buddhi. 2009. Review on thermal energy storage with phase change materials and applications. Renewableand Sustainable Energy Reviews 13(2):318-345.

Tyagi, V. V., and D. Buddhi. 2007. PCM thermal storage in buildings: A state of art. Renewable and Sustainable Energy Reviews 11(6):1146-1166

Yu, S., S. J eong, C. Chyoung, and S. Kim. 2014. Bio-based PCM/ carbon nanomaterials composites with enhanced thermal conductivity. Solar Energy Materials and Solar Cells 120(B): 549-554.

Zhao, C. Y., and G. H. Zhang. 2011 Review on microencapsulated phase change materials (MEPCMs): Fabrication, characterization and applications. Renewable and Sustainable Energy Review. 15(8):3813-3832. ISSN 13640321. 


\section{Acronyms and Abbreviations}

\begin{tabular}{ll} 
Term & Definition \\
ANSI & American National Standards Institute \\
ASA (ALT) & Assistant Secretary of the Army for Acquisition, Logistics, and Technology, \\
ASHRAE & American Society of Heating, Refrigerating, and Air-Conditioning Engineers \\
ASTM & American Society for Testing and Materials \\
ATR & Attenuated Total Reflectance \\
CEERD & U.S. Army Corps of Engineers, Engineer Research and Development Center \\
CERL & Construction Engineering Research Laboratory \\
DrTGA & Derivative of TGA \\
DSC & Differential Scanning Calorimetry \\
ERDC & U.S. Army Engineer Research and Development Center \\
ERDC-CERL & Engineer Research and Development Center, Construction Engineering \\
FTIR & Research Laboratory \\
HQUSACE & Fourier Transform Infrared \\
ISSN & Headquarters, U.S. Army Corps of Engineers \\
MEPCM & International Standard Serial Number \\
MSDS & Microencapsulated Phase Change Material \\
NSN & Material Safety Data Sheet \\
OECIF & National Supply Number \\
OMB & Operational Energy Capital Improvement Fund \\
PCM & Office of Management and Budget \\
SAR & Phase Change Materials \\
SEM & Same As Report \\
SF & Scanning Electron Microscope \\
TGA & Standard Form \\
TR & Thermogravimetric Analysis \\
URL & Technical Report \\
U.S. & Universal Resource Locator \\
& United States \\
\hline
\end{tabular}




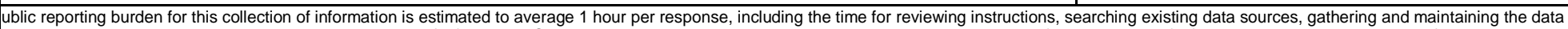

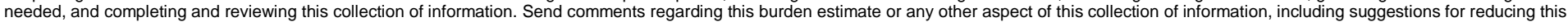

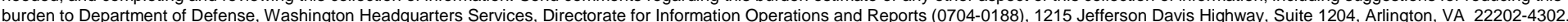

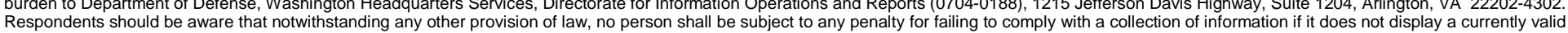
Respondents should be aware that notwithstanding any other provision of law, no person shall
OMB control number. PLEASE DO NOT RETURN YOUR FORM TO THE ABOVE ADDRESS
1. REPORT DATE (DD-MM-YYYY)
21-09-2015
2. REPORT TYPE Final

\section{TITLE AND SUBTITLE}

Simulated Aging and Characterization of Phase Change Materials for Thermal Management of Building Envelopes

\section{AUTHOR(S)}

Elizabeth J . Gao, Jignesh Patel, Veera M. Boddu, L.D. Stephenson, Debbie Lawrence, and Ashok Kumar

3. DATES COVERED (From - To)

\section{5a. CONTRACT NUMBER}

5b. GRANT NUMBER

5c. PROGRAM ELEMENT

5d. PROJECT NUMBER

5e. TASK NUMBER

5f. WORK UNIT NUMBER

7. PERFORMING ORGANIZATION NAME(S) AND ADDRESS(ES)

U.S. Army Engineer Research and Development Center (ERDC)

Construction Engineering Research Laboratory (CERL)

PO Box 9005,

Champaign, IL 61826-9005

8. PERFORMING ORGANIZATION REPORT NUMBER

ERDC/ CERL TR-15-23

\section{SPONSORING I MONITORING AGENCY NAME(S) AND ADDRESS(ES)}

Headquarters, U.S. Army Corps of Engineers (HQUSACE)

$441 \mathrm{G}$ St., NW

Washington, DC 2031405000

10. SPONSOR/MONITOR'S ACRONYM(S)

(1)

11. SPONSOR/MONITOR'S REPORT NUMBER(S)

\section{DISTRIBUTION / AVAILABILITY STATEMENT}

Approved for public release; distribution is unlimited.

\section{SUPPLEMENTARY NOTES}

\section{ABSTRACT}

Because phase change materials (PCMs) can absorb or release heat when they undergo phase changes (solid to liquid, liquid to solid, or solid to gas), they are considered especially promising candidates for use in heating and cooling applications for building envelopes. This work investigated the stability of four commercially available PCMs, and developed an accelerated testing protocol to simulate the long-term performance of PCMs in an operational scenario. The selected PCM s were subjected to up to 5,400 cycles of thermal cycling at 90 minutes per cycle, over wide temperature ranges to simulate 20 years of use in building envelopes. At 3-4 week time intervals, the samples were taken from the thermal cycling chamber and Scanning Electron Microscope (SEM) micrographs, Differential scanning calorimeter (DSC), Thermogravimetric Analysis (TGA), and Fourier Transform Infrared Spectroscopy (FTIR) data were obtained and compared to baseline (pre-cycling data). Results indicated that baseline latent heat values and freezing and melting transition temperatures were generally in accordance with the manufacturer's stated values. All PCM materials evaluated tended to lose some latent heat storage capacity as they underwent thermal cycling.

\section{SUBJECT TERMS}

materials performance, building envelope, energy efficient, energy conservation, phase change materials (PCMs)

\section{SECURITY CLASSIFICATION OF:}

a. REPORT
Unclassified

\section{b. ABSTRACT}

Unclassified

17. LIMITATION
OF ABSTRACT
SAR

18. NUMBER OF PAGES

46 19a. NAME OF RESPONSIBLE PERSON

19b. TELEPHONE NUMBER (include area code) 